\title{
Theoretical Basis of the Mathematical Modeling of Thyristor Converters for Calculation Current and Voltage Harmonics in Power System
}

\author{
Edvard Yunusovich Abdullazyanov ${ }^{1}$, Alexander Ivanovich Fedotov ${ }^{1}$, Sirena Nailevna Zaripova ${ }^{1}$, Evgenii \\ Alexandrovich Fedotov ${ }^{1}$, Natalya Vladimirovna Chernova ${ }^{1} \&$ Georgii Valeriyanovich Vagapov ${ }^{1}$ \\ ${ }^{1}$ Kazan State Power Engineering University, Russian Federation \\ Correspondence: Georgii Valeriyanovich Vagapov, Kazan State Power Engineering University, Russian \\ Federation.
}

Received: December 15, 2014

Accepted: January 22, 2015

Online Published: May 30, 2015

doi:10.5539/mas.v9n6p320

URL: http://dx.doi.org/10.5539/mas.v9n6p320

\begin{abstract}
The problem of developing a mathematically rigorous methods for calculating the composition of the higher harmonics is important in the electrical grids of power systems in modern conditions to increase accountability between power suppliers and its consumers. The first reason is the solution of problems of energy saving. The second reason is delineation of responsibilities to ensure the required power quality between the electricity supply company and customers."
\end{abstract}

Keywords: thyristor converter, mathematical model, power quality

\section{Introduction}

The widespread use in the power conversion devices based power semiconductor materials leads to conducted disturbances in power grids, primarily the higher harmonic components of voltage and current (1-4). Regulating the power quality documents (5-8) set requirements at point of common coupling. Approximation methods of calculation the composition of the higher harmonics have been working successfully up till now. Currently approximation methods are in conflict with the requirements of detail costing of electricity transmission and financial responsibility for ensuring proper power quality between its provider and the consumer.

There is extensive scientific literature of calculations of harmonic voltage and current in power systems. In particular, we can note the monograph (9), which is quite often referred of the authors of publications in this area of scientific exploration. A careful study of published works shows that there are no mathematically rigorous justified equivalent circuit of thyristor converters. Calculations of higher harmonics of current and voltage in power systems almost use one method of accounting for inverter. The composition of the current harmonics thyristor converter is given as a current source in the equivalent circuits of power supply systems. The equivalent circuit as a current source with parallel conductivity given in (9) is postulated without proof and, as will be shown below, incorrect. An uncritical use of methods of specifying the harmonic currents may achieve infinite (theoretically) voltage value in the power system at a resonance harmonics. In fact, the resonance harmonic at the appropriate circuit of the power supply system can be "cut out" from the total spectrum of power converter and currents in the resonant circuit are determined by the magnitude of the voltage source included in the equivalent circuit of the converter. Meanwhile miscalculation of possible level of overvoltage may cause unjustified costs for their elimination.

\section{Method}

2.1 The Equivalent Circuit of Single-Phase Thyristor Converter

Let consider the circuit shown in Figure 1. 


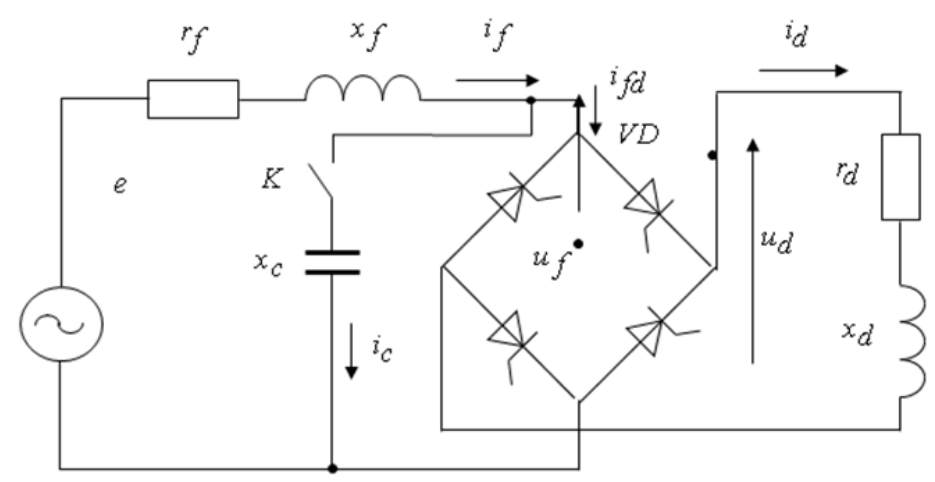

Figure 1. The equivalent circuit of the single-phase inverter.

There are a voltage source e, loaded through the feed line with inductive and active resistance ${ }^{r_{f}},{ }^{x_{f}}$ to the thyristor converter $V D$ with active - inductive load ${ }^{r} d, x_{d}$, the key «K» open. The start time calculation is from zero power at its transition from the negative to the positive values of the area. Let us consider the compilation of differential equations for the arbitrarily selected interval operation of the circuit. Recurrence interval of the converter circuit in angular measure is the radians. At the time of conducting thyristor switch there is short circuit of AC circuits and rectifier load through the transformer $V D$. The condition $\theta=\alpha$ corresponds to start of switching. The condition $i_{d}=\left|i_{f}\right|$ correspond of end of switching. On the switching interval the equation will have the following form $u_{f} \equiv u_{d} \equiv 0, \quad \theta \in[\alpha ; \alpha+\gamma]$ Since the objective is calculation of the transition process on the AC side, the equations that describe the mode of operation of thyristor converter, should be written involving two recurrence intervals of his circuit, which correspond to one period of the power frequency. VD voltage at the inverter side $\mathrm{AC}$ and rectified current linked by the following ratio

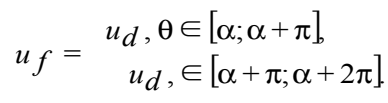

The following expressions use for connecting the phase current and voltage rectifier load during all interval under consideration ${ }^{[\alpha ; \alpha+2 \pi]}$ :

$$
i_{f}=\left\{\begin{array}{l}
i_{d}+i_{\gamma 1} \cdot \Delta K_{1}, i_{\gamma 1}=i_{f}-i_{d}, \\
\Delta K_{1}=K(\theta-\alpha)-K(\theta-\alpha-\gamma) ; \\
-i_{d}+i_{\gamma 2} \cdot \Delta K_{2}, i_{\gamma 2}=i_{f}+i_{d}, \\
\Delta K_{2}=K(\theta-\alpha-\pi)-K(\theta-\alpha-\pi-\gamma) .
\end{array}\right.
$$

where ${ }^{\Delta K_{i}}$ - switching functions.

$K(\theta-\beta)$ are individual functions, that is:

$$
K(\theta-\beta)=\left\{\begin{array}{l}
0, \theta<\beta \\
1, \theta \geq \beta ; \beta=\alpha ; \alpha+\gamma ; \alpha+\pi ; \alpha+\gamma+\pi .
\end{array}\right.
$$

The following boundary conditions are for switching currents $i_{\gamma 1}$ and $i_{\gamma 2}$ in steady state: 


$$
\left.\begin{array}{l}
i_{\gamma 1}(\alpha)=i_{f}(\alpha)-i_{d}(\alpha)=-2 i_{d}(\alpha), \quad i_{\gamma 1}(\alpha+\gamma)=0 \\
i_{\gamma 2}(\alpha+\pi)=i_{f}(\alpha+\pi)+i_{d}(\alpha+\pi)=2 i_{d}(\alpha+\pi), \quad i_{\gamma 2}(\alpha+\gamma+\pi)=0 .
\end{array}\right\}
$$

From (2) we obtain

$$
i_{d}=\left\{\begin{array}{l}
i_{f}-i_{\gamma 1} \cdot \Delta K_{1}, \theta \in[\alpha ; \alpha+\pi] \\
-i_{f}+i_{\gamma 2} \cdot \Delta K_{2}, \theta \in[\alpha+\pi ; \alpha+2 \pi]
\end{array}\right.
$$

Balance voltage equation will have a view from the $\mathrm{AC}$

$$
u_{f}=e-r_{f} i_{f}-x_{f} \frac{d i_{f}}{d \theta}
$$

and from DC

$$
u_{d}=r_{d} i_{d}+x_{d} \frac{d i_{d}}{d \theta}
$$

Combining equation (1), (5) and (7) we get

$$
u_{f}=r_{d} i_{f}+x_{d} \frac{d i_{f}}{d \theta}-r_{d} i_{\gamma 1} \Delta K_{1}-x_{d} \frac{d i \gamma 1}{d \theta} \Delta K_{1}-r_{d} i_{\gamma 2} \Delta K_{2}-x_{d} \frac{d i_{2}}{d \theta} \Delta K_{2}
$$

$F(m, k)$ Such a way we have made the equation of balance of voltage made up by connecting $\mathrm{AC}$ and the rectified voltage inverter the entire change period of line voltage. Local Fourier transform (LFT) (10-12) is used for the transition from the differential equation (8) to the equation for the current and voltage harmonics, which compares the function $f(\theta)$ and its image $F(m, k)$

$$
\begin{gathered}
\stackrel{\&}{F}(m, k)=\frac{2}{h} \int_{\alpha}^{\alpha+h} f(\theta) e^{j k(\theta \quad \alpha)} d \theta \\
n=0 ; \pm 1 ; \pm 2 ; \quad \text { where } \quad k=\frac{2 \pi n}{h} \quad, \quad n=0 ; \pm 1 ; \pm 2
\end{gathered}
$$

For the considered circuit $h=2 \pi$, so $k=n$. In general, LFT allows to write down the desired function as the sum of its harmonics and it is the finite difference (10-12) on the local interval of under consideration. LFT define only complex amplitude of each harmonics in established conditions. LTF is convenient for use, because: integral (9) is used directly for differential equations; algebraic equations compilation regarding harmonics of initial differential equations is formalized. If LTF is used for equation (8), new equation will be in the form of:

$$
\begin{aligned}
& U_{f}(k)=\left(r_{d}+j k x_{d}\right) I_{f}(k) \quad\left(r_{d}+j k x_{d}\right) I_{\gamma}(k) \frac{x_{d}}{\pi}\left[i_{\gamma 1}(\alpha+\gamma) e^{j k \gamma}\right. \\
& \left.i_{\gamma 1}(\alpha)+i_{\gamma 2}(\alpha+\pi+\gamma) e^{j k(\gamma+\pi)} \quad i_{\gamma 2}(\alpha+\pi) e^{j k \pi}\right]
\end{aligned}
$$

There is the notation in equation (10):

$$
I_{\gamma}(k)=\frac{1}{\pi} \int_{\alpha}^{\alpha+\gamma} i_{\gamma 1}(\theta) e^{j k(\theta \quad \alpha)} d \theta+\int_{\alpha+\pi}^{\alpha+\pi+\gamma} i_{\gamma 2}(\theta) e^{j k(\theta \quad \alpha)} d \theta
$$




$$
\left.\left.I_{\gamma}(k)=\frac{1}{\pi} \int_{\alpha}^{\alpha+\gamma} i_{\gamma 1}(\theta) e^{j k(\theta} \alpha\right) d \theta+\int_{\alpha+\pi}^{\alpha+\pi+\gamma} i_{\gamma 2}(\theta) e^{j k(\theta)} \alpha\right) d \theta .
$$

Using LFT for (6) equation we get:

$$
U_{f}(k)=E(k) \quad\left(r_{f}+j k x_{f}\right) I_{f}(k)
$$

Combining equations (10) and (11) into one with boundary condition, we get:

$$
\stackrel{\&}{E}(k)=\left(r_{S}+j k x_{S}\right) \stackrel{\&}{f}_{f}(k) \quad\left(r_{d}+j k x_{d}\right) I_{\gamma}(k) \quad \frac{x_{d}}{\pi}\left[2 i_{d}(\alpha) \quad 2 i_{d}(\alpha) e^{j k \pi}\right]
$$

where $^{r_{S}=r_{f}+r_{d}, x_{S}=x_{f}+x_{d} \text {. Inasmuch as }{ }^{i} \gamma_{1}(\theta)=-i \gamma_{2}(\theta+\pi)}$, so even harmonics will be absent in complex amplitude $I_{\gamma}(k)$. As far as $e(\theta)=-e(\theta+\pi)$ and $i_{f}(\alpha)=-i i_{f}(\alpha+\pi)=-i_{d}(\alpha)$, so all even harmonics will be equal to zero. Therefore, $k$ can take only odd values. Then we obtain

$$
E(k)=\left(r_{S}+j k x_{S}\right) I_{f}(k) \quad\left(r_{d}+j k x_{d}\right) I_{\gamma}(k) \quad \frac{4 x_{d}}{\pi} i_{d}(\alpha)
$$

Analysis of equation (13) show that there are no even harmonics in the harmonic composition of currents and voltages (well-known fact). However a combination of a current source and voltage source are new, which is shown by the calculate equivalent circuit in Figure 2, where $E_{d}=4 x_{d}{ }^{i}(\alpha) / \pi$.

We compare results of calculation harmonics on side of $\mathrm{AC}$ by demonstrated calculated equivalent circuit and by classical methodic, based on integrating the instantaneous values of current. The transition process for the circuit in Figure 1 upon conditions ${ }^{r}=0$ and ${ }^{x} f=0$ has been considered in (5). Current change on the first interval of repeatability $\theta \in[\alpha ; \alpha+\pi]$ in the inverter load stepped coordinate system in the steady is stated by the law:

$$
i_{d}^{(1)}=i_{f}=\frac{E}{z_{d}} \sin \left(\theta-\varphi_{d}\right)+\left[i_{d}(\alpha)-\frac{E}{z_{d}} \sin \left(\alpha-\varphi_{d}\right)\right] e^{-(\theta-\alpha) \xi_{d}}
$$

where $z_{d}=\sqrt{r_{d}^{2}+x_{d}^{2}}, \quad \xi_{d}^{-1}=\operatorname{tg} \varphi_{d}=x_{d} / r_{d}$.

current change by the law:

$$
i_{d}^{(2)}=i_{f}=\frac{E}{z_{d}} \sin \left(\begin{array}{lll}
\theta & \varphi_{d}
\end{array}\right) \quad i_{d}(\alpha) \quad \frac{E}{z_{d}} \sin \left(\begin{array}{lll}
\alpha & \varphi_{d}
\end{array}\right) e^{\left(\begin{array}{lll}
\theta & \alpha & \pi
\end{array}\right) \xi}
$$

on the second interval, when $\theta \in[\alpha+\pi ; \alpha+2 \pi]$.

Next phase is calculation of higher harmonics $(k \neq \pm 1)$ of current in the network in a complex form. After integration of current ${ }^{i_{f}}$ according to (14) and (15) equation we get: 


$$
I_{f}(k)=\frac{2 x_{d}}{\pi} i_{d}(\alpha) \frac{E}{z_{d}} \sin \left(\alpha \quad \varphi_{d}\right) \frac{1+e^{\pi \xi}}{r_{d}+j k x_{d}}
$$

Equation (17) can be simplified as follows. Equation (14) can be used for this, where $\theta=\alpha+\pi$. After minor changes equation looks like a (5):

$$
-\frac{E}{z_{d}} \sin \left(\theta-\varphi_{d}\right)=i_{d}(\alpha) \frac{1-e^{-\pi \xi_{d}}}{1+e^{-\pi \xi_{d}}} .
$$

Substituting the (18) in (17) the final equation we come to:

$$
I_{f}(k)=\frac{2 x_{d}}{\pi} i_{d}(\alpha)+i_{d}(\alpha) \frac{1 e^{\pi \xi}}{1+e^{\pi \xi}} \frac{1+e^{\pi \xi}}{r_{d}+j k x_{d}}=\frac{4 x_{d} i_{d}(\alpha)}{\pi\left(r_{d}+j k x_{d}\right)} .
$$

Comparison of equations (13) and (19) give the same result under such initial phase conditions $\left(r_{s}=r_{d}, x_{s}=x_{d}, E(k)=0, k \neq \pm 1\right)$. Received mathematical model, Figure 2, is reduced to well-known (1), if we assume ${ }^{x_{d} \rightarrow \infty}$ as a basis. It is easy to show that the converter circuit may be reduced to a current source ${ }^{J(n)}$ with included parallel conductivity $y(n)$ :

$$
\begin{array}{r}
J(n)=J_{\gamma}(n)+\frac{4}{\pi} \frac{x_{d} i_{d}(\alpha)}{r_{d}+j n x_{d}}, \quad y(n) \quad 1=r_{d}+j n x_{d} . \\
J(n)=J_{\gamma}(n)+\frac{4}{\pi} \frac{x_{d}{ }^{i}(\alpha)}{r_{d}+j n x_{d}}, \quad y(n) \quad 1=r_{d}+j n x_{d} .
\end{array}
$$

Rectified current completely is flattened $\left({ }^{i} d(\theta)=I_{d}=\right.$ const $)$, if ${ }^{x_{d} \rightarrow \infty}$ and

$$
\stackrel{\&}{J}(n)=\stackrel{\&}{J}(n)+\frac{4}{\pi} \frac{I_{d}}{j n x_{d}}, \quad \stackrel{\&}{y}(n) \rightarrow 0 .
$$

Equation (20) conforms to well-known composition of harmonics in phase current of converter with condition of completely rectified current. Comparison with (9) shows, that current sources coincide only in a special case of application of equation (20), when $x_{d} \rightarrow \infty$. So, developed equivalent circuit is mathematically strict. It can be used in any circuit an external network. There is only one admission about an external network that has not been directly used in the derivation of the equivalent circuit. It is voltage form e which guarantees two commutations for the one period. It can be assumed that the mathematical model of thyristor converter is received in a general form, considering that the condition is implemented in industrial electrical networks .Let us compare precision of the calculation electricity losses from the higher harmonic components realize for a given form of rectified current and an equivalent circuit, Figure 2.

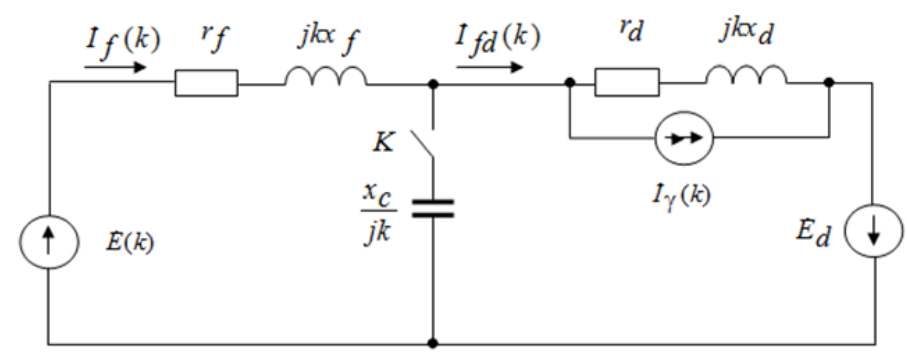

Figure 2. The equivalent circuit of the electrical network for the k-th harmonic. 
$x_{f}=0$ is a basis and allows to switch thyristors instantaneous by. AC Harmonics of completely rectified current are calculated by next equation:

$$
I_{f}(k)=\frac{4 I_{d}}{\pi k} \Rightarrow \quad I_{*} f(k)=\frac{4}{\pi k}
$$

As far as completely rectified current equals to

$$
\begin{gathered}
I_{d}(0)=\frac{1}{\pi} \int_{\alpha}^{\alpha+\pi} i_{d} d \theta=\frac{2}{\pi} \frac{E}{z_{S}} \cos \left(\varphi_{S}-\alpha\right)+\frac{2}{\pi} \frac{E}{z_{s} \xi_{S}} \sin \left(\varphi_{S}-\alpha\right)=\frac{2}{\pi} \frac{E}{r_{S}} \cos \alpha \\
I_{d}(0)=\frac{1}{\pi} \int_{\alpha}^{\alpha+\pi} i_{d} d \theta=\frac{2}{\pi} \frac{E}{z_{S}} \cos \left(\varphi_{S}-\alpha\right)+\frac{2}{\pi} \frac{E}{z_{s} \xi_{S}} \sin \left(\varphi_{S}-\alpha\right)=\frac{2}{\pi} \frac{E}{r_{S}} \cos \alpha \\
\text { where }{ }_{s}=\sqrt{r_{S}^{2}+x_{d}^{2}}, \quad \xi_{S}=r_{S} / x_{d}=\chi_{S}^{-1}, \quad \operatorname{tg} \varphi_{s}=\chi_{s},
\end{gathered}
$$

using the equation (13) we get in the general case:

$$
\frac{I_{f}(k)}{I_{d}(0)}=I_{*}^{\prime \prime} f(k)=\frac{4}{\pi \sqrt{\left(\xi_{S}\right)^{2}+k^{2}}} \frac{i_{d}(\alpha)}{I_{d}(0)}
$$

At the same time

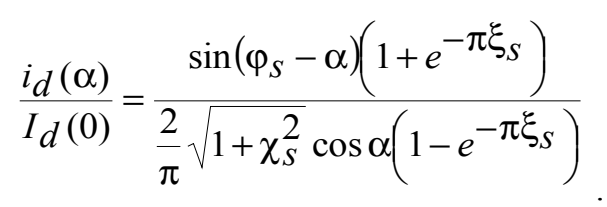

The boundary of continuous current mode is the following condition to control angle: ${ }^{\alpha=} \varphi_{S}$ Figure 3 shows example of graphs of the third harmonic phase current calculated by the equation (22).

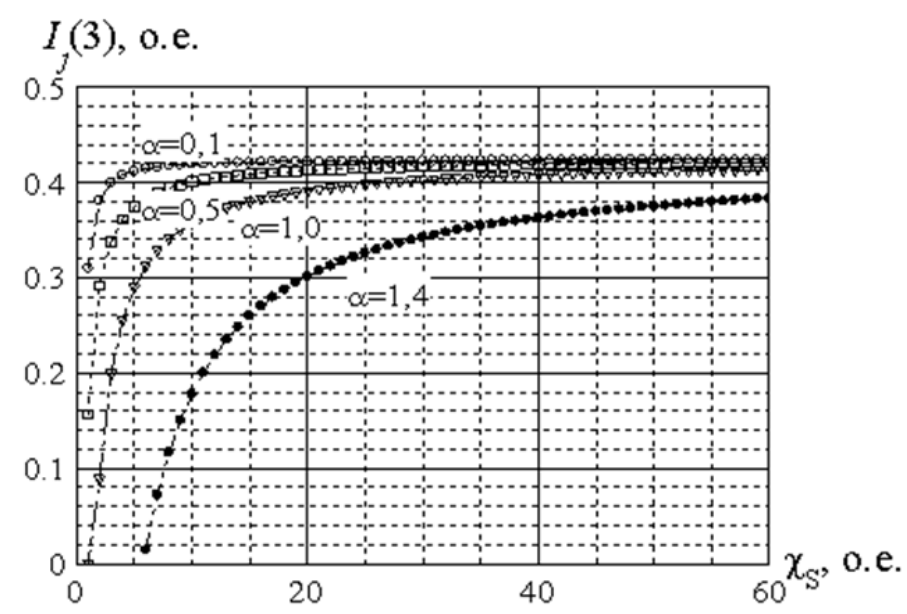

Figure 3. Graphs of the third harmonic current

They clearly show that the equation (21) can be used only within a certain range of the changes: smaller $\chi_{s}$ and the more ${ }^{\alpha}$, the greater the error in the calculation of equation (21). The same results will be for higher numbers of harmonics. Loss of power is proportional to the current square. Figure 4 shows the dependence on error calculation $(k)$, received by the equation: 


$$
\delta(k)=\left\{\left(I_{*} c(k)\right)^{2}-\left(I_{*}{ }_{c}(k)\right)^{2}\right\}\left(I_{*}^{\prime \prime} c(k)\right)^{-2}
$$

Analysis of the results shows that, even at $\chi_{s}<20$ error of the power loss by current (21) becomes unacceptably higher. Methods that give higher value of energy losses (it is obtained in this case) are not good, the same situation is with methods giving underestimated value of energy losses.

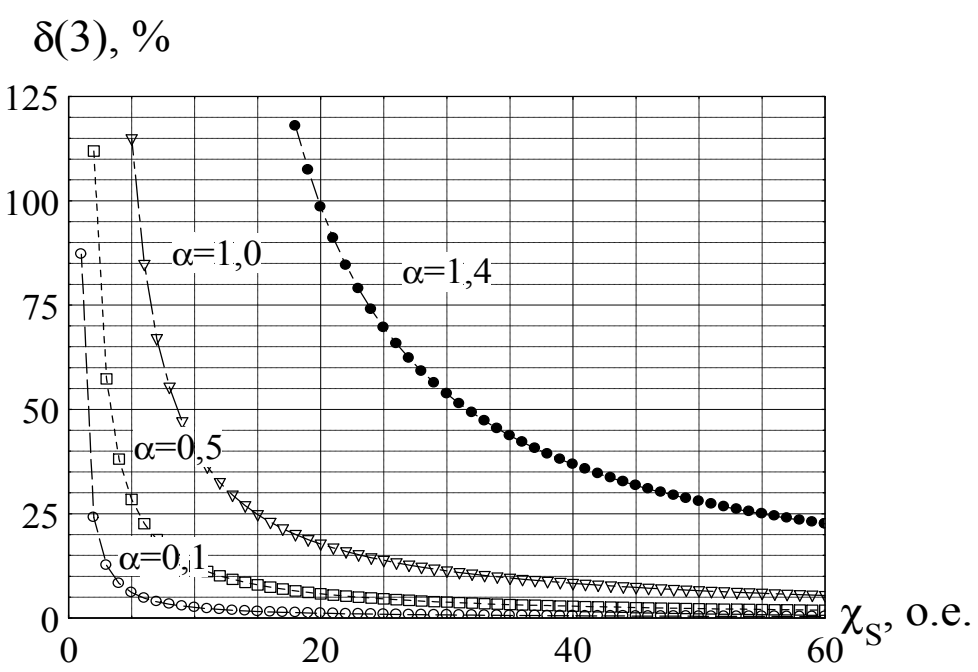

Figure 4. Graphs of the relative error in the determination of losses Power for the third harmonic current

There is no possibility to normalize permissible level of energy losses when calculation level of energy losses is higher. In such a way, when the real level of energy losses coincide with calculation level of energy losses, then may be a situation without necessity of activities to reduce them. But in fact level of energy losses from higher harmonics must be lower and search for causes of increasing of energy losses is extremely necessary.

\subsection{Inverter Work in the Network with Resonant Circuit}

Consideration of the modes of operation thyristor converters in isolation from the operation of the system of power supply (SPS) is prevalent technique for calculating the power losses from higher harmonics. Sole, accounting of changes in existing supply voltage converters is executed at change load of the system of power supply or at, also, voltage is changed from side of the electricity supplier. After determining the composition of the current harmonics on the input of each thyristor converter we implement the application of harmonics to the all of system of power supply and then we calculate the resulting impact the harmonics composition on the power quality parameters (PQP). Let us consider this case as the wrong theoretical premises can lead to unjustified decisions in limiting the surge in electrical power system (EPS), where there are resonant phenomenon.

The latter can be triggered by the presence of condenser facilities, which serve to enhance the cosine of the angle of the load or voltage regulation, and the distributed capacitance of the network elements of the EPS. In any case, it is necessary to prevent dangerous overvoltage's resonance must have possibility their correct calculation. Figure 1 shows a typical example of the circuit which reduces EPS. The first condition of the circuit in Figure 1, when the key «K» closed.

There are installed capacitors on the tires $0.4 \mathrm{kV}$, and the converter is powered by $0.4 \mathrm{~kW}$ on a short line. Peculiarity of mode of the resulting circuit is a break of the current ${ }^{i} f d$ in moment of switching thyristors at $\theta=\alpha$ and $\theta=\alpha+\pi$. Equations of balance of voltage will be: 


$$
\begin{gathered}
u_{f}=\left\{\begin{array}{l}
r_{d} i_{d}+x_{d} \frac{d i_{d}}{d \theta}=\left(r_{d} i_{f d}+x_{d} \frac{d i f d}{d \theta}\right) \Delta K_{3}-r_{d} i_{\gamma 1} \Delta K_{1}-x_{d} \frac{d i_{\gamma 1}}{d \theta} \Delta K_{1}, \\
-r_{d} i_{d}-x_{d} \frac{d i_{d}}{d \theta}=\left(r_{d}{ }_{f d}+x_{d} \frac{d i f d}{d \theta}\right) \Delta K_{4}-r_{d} i_{2} \Delta K_{2}-x_{d} \frac{d i \gamma_{2}}{d \theta} \Delta K_{2},
\end{array}\right. \\
u_{f}=\left\{\begin{array}{l}
r_{d}{ }^{i} d+x_{d} \frac{d i_{d}}{d \theta}=\left(r_{d}{ }^{i} f d+x_{d} \frac{d i f d}{d \theta}\right) \Delta K_{3}-r_{d} i_{\gamma 1} \Delta K_{1}-x_{d} \frac{d i_{\gamma 1}}{d \theta} \Delta K_{1}, \\
-r_{d} i_{d}-x_{d} \frac{d i_{d}}{d \theta}=\left(r_{d}{ }^{i} f d+x_{d} \frac{d i f d}{d \theta}\right) \Delta K_{4}-r_{d} i_{2} \Delta K_{2}-x_{d} \frac{d i \gamma 2}{d \theta} \Delta K_{2},
\end{array}\right.
\end{gathered}
$$

where

$$
\begin{aligned}
& \Delta K_{3}=K(\theta-\alpha-\varepsilon)-K(\theta-\alpha-\pi+\varepsilon) ; \\
& \Delta K_{4}=K(\theta-\alpha-\pi-\varepsilon)-K(\theta-\alpha-2 \pi+\varepsilon), \quad \varepsilon \rightarrow 0 .
\end{aligned}
$$

Another view

$$
\begin{aligned}
& u_{f}=\left(r_{d}{ }^{i} f d+x_{d} \frac{d i f d}{d \theta}\right)\left(\Delta K_{3}+\Delta K_{4}\right)- \\
& -r_{d} i_{\gamma 1} \Delta K_{1}-x_{d} \frac{d i_{\gamma 1}}{d \theta} \Delta K_{1}-r_{d} i_{\gamma 2} \Delta K_{2}-x_{d} \frac{d i_{2}}{d \theta} \Delta K_{2} .
\end{aligned}
$$

Let us use LTF for equation (24). In view of the following conditions ${ }^{i} f d(\alpha+\varepsilon)=-i f d(\alpha+\pi+\varepsilon)$ and $i_{f d}(\alpha+\pi-\varepsilon)=-i_{f d}(\alpha+2 \pi-\varepsilon)=i_{d}(\alpha)$ are satisfied in the steady state at $\varepsilon \rightarrow 0$ and for odd values of the $\mathrm{k}$ we get

$$
U_{f}(k)=\left(r_{d}+j k x_{d}\right) I_{f d}(k) \quad\left(r_{d}+j k x_{d}\right) I_{\gamma}(k) \frac{4 x_{d}}{\pi} i_{d}(\alpha)
$$

$n x_{f}=x_{c} / n$ From equation (25) it is ensue converter equivalent circuit may be use, Figure 2, but with the inclusion of a capacitance branch (key «K» closed). Let us now consider resonance mode at a harmonic current. Let is assume that $r_{f}=0, E(k)=0$ at $k \neq \pm 1$ and for $k=n$ satisfies the condition $n x_{f}=x_{c} / n$ As much as

$$
I_{f d}(k)=\frac{4}{\pi} x_{d} i_{d}(\alpha) \times j k x_{f} \frac{x_{c}}{j k} j k x_{f}+\frac{x_{c}}{j k} \quad{ }^{1}+r_{d}+j k x_{d},
$$

then for $\mathrm{k}=\mathrm{n}$ will be

$$
I_{f d}(n)=\frac{4}{\pi} x_{d} i_{d}(\alpha)\left(\infty+r_{d}+j n x_{d}\right) 1=0 .
$$

Misunderstandings with endless voltage at the resonant frequency $k=n$ are explained by fact that it is shows in (26) and at the same time ${ }^{x_{d} \rightarrow \infty}$. 
Then it turns to uncertain expression of the form $\infty / \infty$.

Disclosure of undefined expressions is arbitrarily associated with preservation in the phase current harmonic spectrum of the resonant frequency (14). From the resulting equivalent circuit it follows the voltage on the resonant circuit is determined by a voltage source in combination to the current source switch.

\subsection{Example}

The numerical experiment performed in Mathcad for the case of resonance tuning filter ${ }^{L_{f}-C_{c}}$ on 3-rd harmonic. Figure 4 shows a the current waveform ${ }^{i} f d$ at the following initial data: $E=10 \mathrm{~V},{ }^{x}=\pi^{*} 0,1 \quad$ Om;

$$
r_{d}=1 \mathrm{Om} ; x_{d}=\pi * 10 \mathrm{Om} ; x_{c}=(\pi * 0,112579092936)^{-1} \text { Om. }
$$

Thyristor of converter is taken completely open, that is, working as diodes. The calculation of the spectrum of the current and voltage showed the absence of the third current harmonic and the presence of the third voltage harmonic. Figure 5 shows a waveform of switching current of the selected phase current.

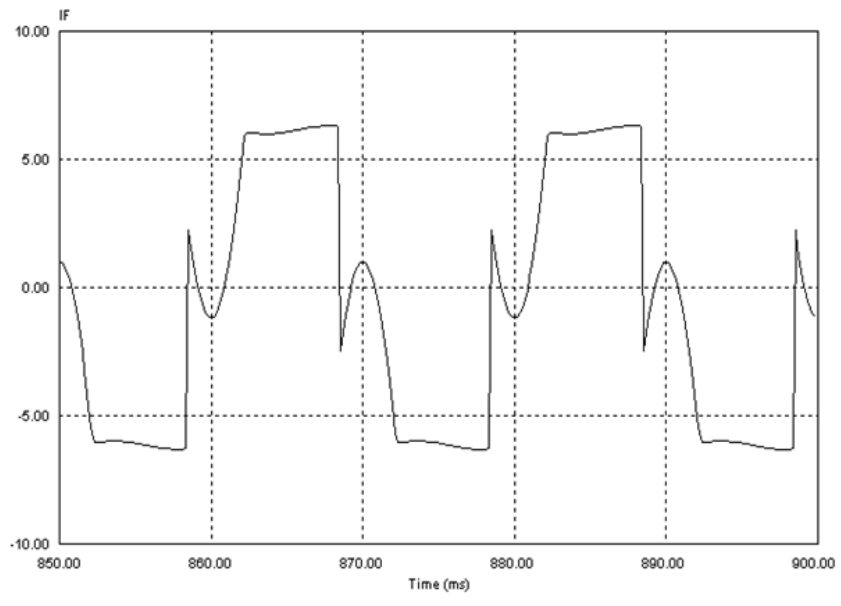

Figure 5. Phase current at the converter input, $\mathrm{n}=3$

Calibration calculation has been performed by determining the voltage at the resonance capacitance harmonic $n$

$=3$ for the equivalent circuit in Figure 2. according to the mathematical oscillograms of currents in Figure 5 and

6. The third harmonic ${ }^{i}{ } 3$ of the switching current ${ }^{i_{\gamma}}$ is obtained by its Fourier expansion.

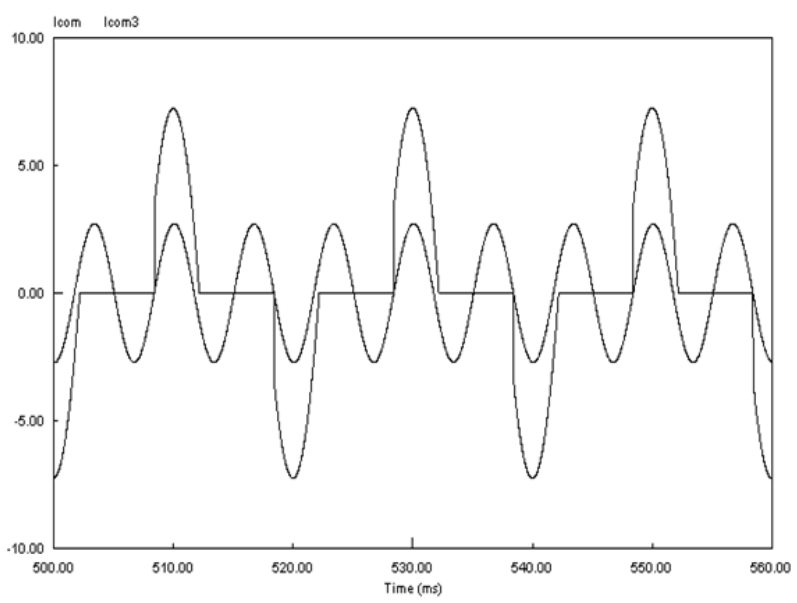

Figure 6. Switching current and its third harmonic 
Figure 6 shows a vector diagram for the harmonic of triple frequencies, built in accordance with the phase current, which in relation to the origin is behind by 180 degrees.

Considering that in PSL sinusoidal component of the complex amplitude as compared with the usual complex representation of the Fourier series is rotated 180 degrees, it can be written

$$
I_{\gamma}(3)=j I_{\gamma}(3) .
$$

There are

$$
\begin{aligned}
& \stackrel{\&}{U}_{f}(3)=\stackrel{\&}{U}_{d \alpha} \quad\left(r_{d}+j k x_{d}\right) \stackrel{\&}{I}_{\gamma}(3)=\frac{4}{\pi} x_{d} i_{d}(\alpha) \quad j r_{d} I_{\gamma}(3)+3 x_{d} I_{\gamma}(3) \\
& \stackrel{\&}{U}_{f}(3)=\stackrel{\&}{U}_{d \alpha} \quad\left(r_{d}+j k x_{d}\right) \stackrel{\&}{I_{\gamma}(3)}=\frac{4}{\pi} x_{d} i_{d}(\alpha) \quad j r_{d} I_{\gamma}(3)+3 x_{d} I_{\gamma}(3) \\
& U_{d \alpha}=\frac{4}{\pi} x_{d} i_{d}(\alpha) ; \quad \stackrel{\&}{E}{ }_{\gamma \mathrm{a}}=3 x_{d} I_{\gamma}(3) ; \quad \stackrel{\&}{E_{\gamma \mathrm{a}}}=3 x_{\mathrm{H}} I_{\gamma 3}+\left(U_{d \alpha}\right) ; \\
& \stackrel{\&}{\Delta} \underset{\gamma p}{ }=j r_{d} I_{\gamma}(3) ; \quad \stackrel{\&}{U_{c}}=\Delta E_{\gamma \mathrm{a}}+\Delta E_{\gamma p}^{\&} \text {. } \\
& U_{d \alpha}=\frac{4}{\pi} x_{d} i_{d}(\alpha) ; \quad \stackrel{\&}{E} \gamma_{\mathrm{a}}=3 x_{d} I_{\gamma}(3) ; \quad \stackrel{\&}{E_{\gamma \mathrm{a}}}=3 x_{\mathrm{H}} I_{\gamma 3}+\left(U_{d \alpha}\right) ;
\end{aligned}
$$

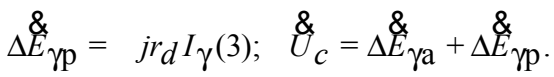

It is determined by the oscilloscope traces in Figure 5 and Figure6, that ${ }^{i}{ }_{\mathrm{H}}(\alpha)=6,29 \mathrm{~A} ;{ }^{I} \gamma_{3}=2,73 \mathrm{~A}$. The result is the following, Figure 7:

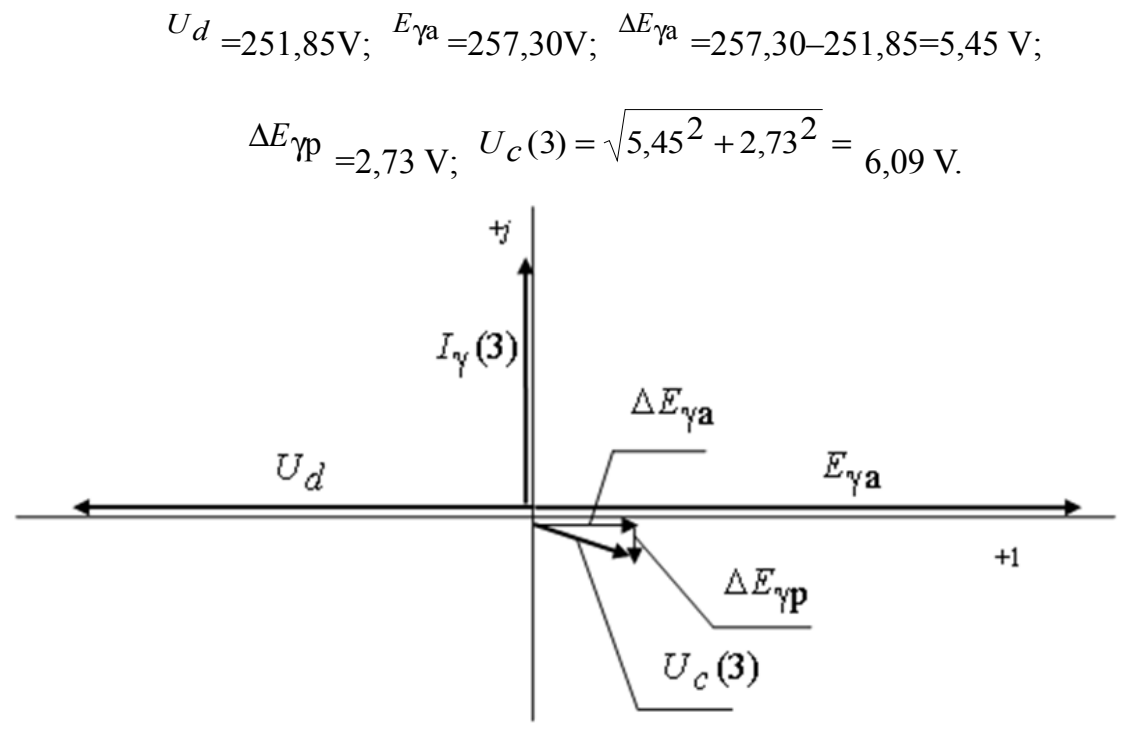

Figure 7. Vector diagram of voltages at the resonant harmonic

It was defined from the calculation of the spectrum: $U_{c}(3)=6,00 \mathrm{~V}$. The error is $1.5 \%$ in the calculation of the equivalent circuit of Figure 2 and related with the definition of the currents on the oscilloscope traces.

The phase shift $\alpha$ of the natural opening of the anode of thyristor is: if $n=3 \alpha=-1,5 \mathrm{~ms}$; if $n=5 \alpha=-0,27 \mathrm{~ms}$; if $n=7 \alpha=0,28 \mathrm{~ms}$. Accordingly, the duration of switching was: $3.83 \mathrm{~ms}(\mathrm{n}=3), 2.82 \mathrm{~ms}(\mathrm{n}=5)$ and $2.22 \mathrm{~ms}(\mathrm{n}=7)$. The calculation results show that a small change of rectified current (within a few percent with the transition from the $\mathrm{n}=3$ to the mode for $\mathrm{n}=5$ ), the commutation angle has changed by more than $25 \%$. Therefore, the phase shift of the start switch is critical to the composition of harmonic. Thus there is a change in the switching current form at one and the same law of his change. Simultaneous change in the duration of switching leads to the exclusion of the resonant harmonics of the phase current load at almost completely smoothed rectified 
current. In such a way, as shown by research, the network of power supply system has a direct impact on changes in the inverter, which, in turn by, its composition of harmonics is influenced the PQP in electricity supply system. It is obvious that the approach to thyristor converters as to independent elements of power supply system contain methodological error. Calculation mode converter in determining with the composition of the current harmonics should be associated to the parameters of power supply system, only then reliable results be obtain by PQP and overvoltage resonant harmonics. We assume that the rectified current is completely flattened. The switching current is determined only by value of the rectified current and predetermined control angle with the application of sinusoidal voltage (16). Have in mind that the electricity network has no effect on the rectified current and the switching current, and respectively on the composition of the phase current harmonics as well. The postulate of the full smoothed rectified current becomes mathematical strict when the inductance of rectifier load is $x_{d} \rightarrow \infty$

It is clear that physically realizable values $x_{d}$ are always finite. Therefore it is important to identify for which loads such an "ideal" case can be considered without introducing appreciable errors in calculations. Winding data of DC motors and associated oscillograms confirm that in excitation circuits the pulsation of the rectified current are negligible, so there is entirely reasonable to assume $x_{d} \rightarrow \infty$ However, in circuits of the armature winding inductance is an order of magnitude less than in the excitation circuits. Under unfavorable combinations of engine output, its load and its angle of control can go into a mode of discontinuous currents in general. We examined an example of a change in the current switching and "cutting" harmonic resonance in phase current converter occurred due to network parameters. Thus if the rectified current form is specified beforehand by the anchor chain, then it is possible in some cases to get results that differ from reality. The presence of current smoothing reactors can be considered one of the arguments in favor of the above-mentioned postulate. But when they are installed it is wrong to assume that $x_{d} \rightarrow \infty$. Since the greater their inductance, the slower the working up of motor perturbations. For example, an automatic control system for production technology requirements must support constant engine speed under strictly defined limits. The task is the development of equivalent circuits of three-phase converters, which directly, and not indirectly, take into account load parameters and the power supply system network, which can be estimated as more difficult in comparison with the task of developing a single-phase equivalent circuit managed converter. Operating modes of single-phase and three-phase controlled converters have one fundamental difference (when the three-phase inverter is in native mode - sequential conductivity of two and three thyristors): at specified time intervals there is no electrical connection between the supply network phase and three-phase rectifier load converter, and thus the phase current is interrupted. In a single-phase converter intermittent current is possible only when there is a certain value of the EMF, counter included in the circuit of the active-inductive load.

\subsection{Initial Equations of Equivalent Circuit for Bringing a Three-Phase Thyristor Converter to One Phase}

Let us consider a three-phase controlled thyristor converter connected to the active - inductive load and source of EMF, Figure8.

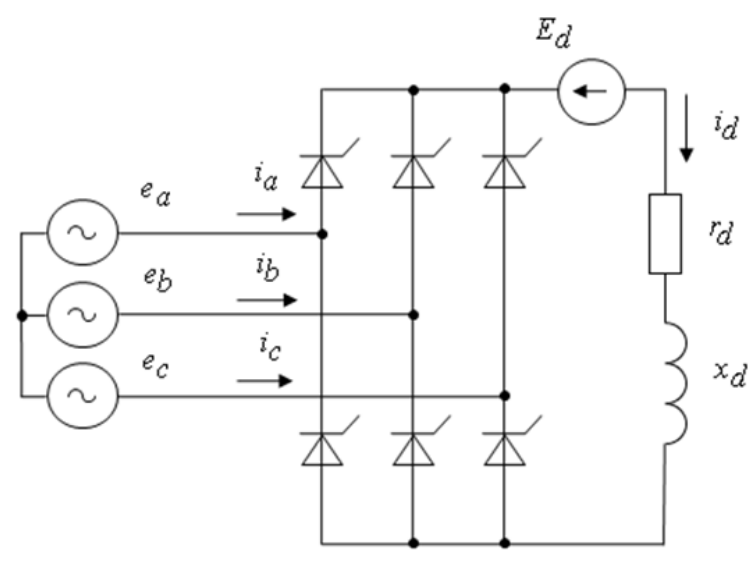

Figure 8. Circuit diagram of the thyristor converter

This load corresponds to the connection to a DC motor. Accept that the a main power supply of infinite power, which allows instantaneous switching processes. This is done to more precisely identify the features 
making up the mathematical model of the object of research. Below we will take into account the final duration of the switching, when due to inductance in the power circuits of converter (inductance network or converting transformer) the switch conductive phase is delayed. We assume, as before, that the thyristor of converter is ideal, and control angle $\alpha$ is measured from the zero value of the corresponding phase of EMF.

The recurrence interval of the converter is $\pi / 3$ to the side of the rectified current; in AC the duration of positive and negative values of each phase current is $2 \pi / 3$ Phase current is missing during the two intervals, each of duration $\pi / 3$ when the appropriate thyristor of converter closes (17). Since the task is determining the harmonics on the AC side, it is necessary to describe the modes of operation of the converter during the entire period of industrial frequency (18-20).

The equation of the voltage balance relative to the phase «a» has the forms:

$$
\left.\begin{array}{ll}
e_{a}-e_{b}=r_{d} \cdot i_{d}+x_{d} \frac{d i_{d}}{d \theta}+E_{d}, & \theta \in[\alpha ; \alpha+\pi / 3] ; \\
e_{a}-e_{b}=-r_{d} \cdot i_{d}-x_{d} \frac{d i_{d}}{d \theta}-E_{d}, & \theta \in[\alpha+\pi ; \alpha+4 \pi / 3] ; \\
e_{a}-e_{c}=r_{d} \cdot i_{d}+x_{d} \frac{d i_{d}}{d \theta}+E_{d}, & \theta \in[\alpha+\pi / 3 ; \alpha+2 \pi / 3] ; \\
e_{a}-e_{c}=-r_{d} \cdot i_{d}-x_{d} \frac{d i_{d}}{d \theta}-E_{d}, & \theta \in[\alpha+4 \pi / 3 ; \alpha+5 \pi / 3] .
\end{array}\right\}
$$

Given that $i_{a}=i_{d}$ at $\theta \in[\alpha ; \alpha+2 \pi / 3]$ and $i_{a}=-i_{d}$ at $\theta \in[\alpha+\pi ; \alpha+5 \pi / 3]$ the equation (27) can be reduced to the following form

$$
\begin{gathered}
e_{a}-e_{b}= \begin{cases}r_{d} \cdot i_{a}+x_{d} \frac{d i_{a}}{d \theta}+E_{d}, & \theta \in[\alpha ; \alpha+\pi / 3] ; \\
r_{d} \cdot i_{a}+x_{d} \frac{d i_{a}}{d \theta}-E_{d}, & \theta \in[\alpha+\pi ; \alpha+4 \pi / 3] ;\end{cases} \\
e_{a}-e_{c}= \begin{cases}r_{d} \cdot i_{a}+x_{d} \frac{d i_{a}}{d \theta}+E_{d}, & \theta \in[\alpha+\pi / 3 ; \alpha+2 \pi / 3] ; \\
r_{d} \cdot i_{a}+x_{d} \frac{d i_{a}}{d \theta}-E_{d}, & \theta \in[\alpha+4 \pi / 3 ; \alpha+5 \pi / 3] .\end{cases}
\end{gathered}
$$

For three-phase electrical circuits it is accepted that the equivalent circuit reduces to one phase. Usually reduction is performed in phase " $a$ ". If we combine equations (28) and (29) into one with switching coefficients we get:

$$
K_{a}(\theta) e_{a}-K_{a b}(\theta) e_{b}-K_{a c}(\theta) e_{c}=K_{a}(\theta)\left[r_{d} i_{a}+x_{d} \frac{d i_{a}}{d \theta}\right]+K_{e}(\theta) E_{d}
$$

Functional dependencies of switching coefficients $K_{a}(\theta), K_{a b}(\theta), K_{a c}(\theta), K_{e}(\theta)$ are shown in Figure 9. We add addendum $K_{a a}(\theta) \cdot e_{a}$ on the left and right parts of equation (30) (see graph for $K_{a a}(\theta)$ Figure 9). 


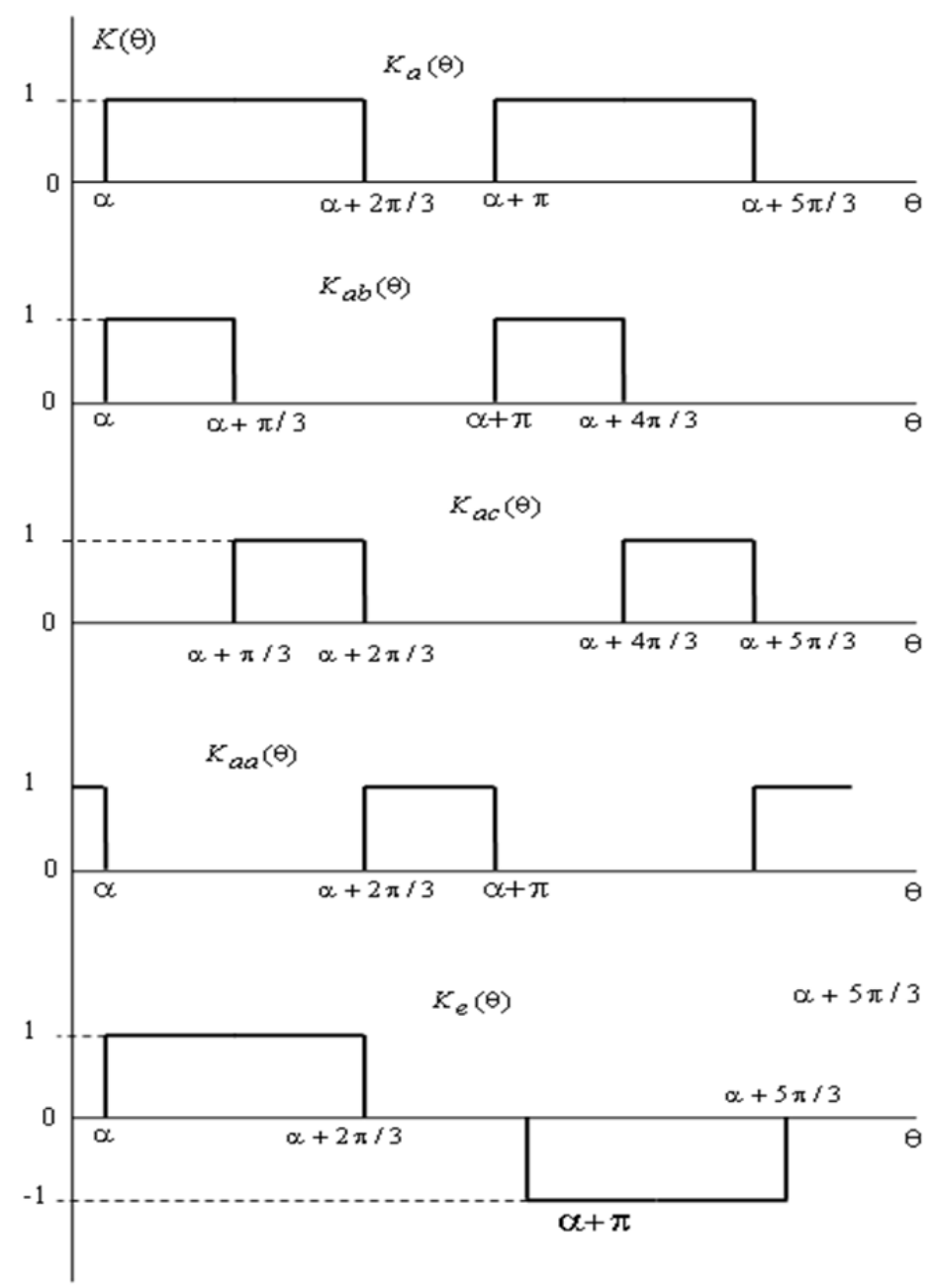

Figure 9. Graphics of switching functions

As a result, EMF phase "a" enters into the equation for the whole period of industrial frequency. This procedure is necessary for the correlation of the converter's equivalent circuit with the substitution circuits of the rest of the electric power supply system. As a result, equation (30) takes the following form:

$$
e_{a}=K_{a}(\theta)\left[r_{d} i_{a}+x_{d} \frac{d i_{a}}{d \theta}\right]+\delta e_{a},
$$

where

$$
\delta e_{a}=K_{a a}(\theta) e_{a}+K_{a b}(\theta) e_{b}+K_{a c}(\theta) e_{c}+K_{e}(\theta) E_{d}=\delta(\theta) e_{s}+K_{e}(\theta) E_{d}
$$

which we call an EMF distortion. At first glance, this expression looks unpromising for further manipulation, because it initially contains the EMF $\delta e_{a}$, which in a real power system transforms into a voltage source $\delta u_{a}$. The voltage, first, is not determined yet, and second, the supply voltage will contain higher harmonics caused by the work of the converter itself, which also has not yet been determined. But analysis of the possibility of using equation (31) for calculating the harmonic components leads to the following conclusions:

1. There are no principal obstacles to the integration of the resulting equivalent of circuit converter in the equivalent circuit of an arbitrarily complex system of power supply, since at the input terminals relative to EMF

$e_{a}$ provide reproduction modes of operation converter during the whole period of supply voltage frequency.

2. Nonlinearity modeling object shows up in the fact that EMF distortion depends on the mode of operation of the entire power supply system because this is a single-phase EMF "cutting" of pieces of three-phase of supply voltage.

Formally this fact is not allowed to combine into the equivalent circuit of converter and the rest of the power 
supply system. But the use of the method of successive approximations reduces the problem of harmonic calculation to a linear.

3. When the converter is in the power supply system (PSS), the impact of EMF distortion $\delta e_{a}$ on the quality of the voltage will depend on the parameters of their own PSS and the parameters of the load from the side of rectified voltage.

Therefore, the approximate determination of the spectrum $\delta e_{a}$, excluding the higher harmonics of voltage $u_{a}$ may not have any effect on the results of calculations, if the degree of contribution of EMF distortion $\delta e_{a}$ harmonics of the phase current is insignificant.

\subsection{Equivalent Circuit of a Three-Phase Inverter for Harmonic Calculation Excluding Switching Processes}

In order to go to the actual equivalent circuit we use local Fourier transform (LFT) for equation (31), assuming that $h=2 \pi$ :

$$
\begin{gathered}
\frac{2}{2 \pi} \int_{\alpha}^{\alpha+2 \pi} e_{a} \cdot e^{-j k(\theta-\alpha)} d \theta=\frac{2}{2 \pi} \int_{\alpha}^{\alpha+2 \pi} K_{a}(\theta) \cdot\left(r_{d} \cdot i_{a}+x_{d} \frac{d i_{a}}{d \theta}\right) \times \\
\times e^{-j k(\theta-\alpha)} d \theta+\frac{2}{2 \pi} \int_{\alpha}^{\alpha+2 \pi} \delta e_{a} \cdot e^{-j k(\theta-\alpha)} d \theta .
\end{gathered}
$$

As a result of integration we obtain (phase indexes "a" left out):

$$
\begin{gathered}
E(k)=\left(r_{d}+j k x_{d}\right) \cdot I(k)+\delta \dot{E}(k)+\frac{x_{d}}{\pi} \times \\
\times\left[i(\alpha+2 \pi / 3) \cdot e^{-j k 2 \pi / 3}-i(\alpha)+i(\alpha+5 \pi / 3) \cdot e^{-j k 5 \pi / 3}-i(\alpha+\pi) \cdot e^{-j k \pi}\right] .
\end{gathered}
$$

In steady state operation it will be

$$
i(\alpha)=i(\alpha+2 \pi / 3)=-i(\alpha+\pi)==-i(\alpha+5 \pi / 3)=i_{d}(\alpha) .
$$

Then

$$
E(k)=\left(r_{d}+j k x_{d}\right) \cdot \dot{I}(k)-\frac{x_{d}}{\pi} i_{d}(\alpha) \cdot\left(1-e^{-j k \pi}\right) \cdot\left(1-e^{-j k 2 \pi / 3}\right)+\delta E(k) .
$$

From equation (32) it follows that there is an absence of even harmonics and of harmonics with a multiple of three. For the other odd harmonics we can write:

$$
\dot{E}(k)=\left(r_{d}+j k x_{d}\right) \cdot \dot{I}(k)-\frac{2 x_{d}}{\pi} \cdot \dot{a}(k) \cdot i_{d}(\alpha)+\delta \dot{E}(k),
$$

Where $a(k)=1-e^{-j k 2 \pi / 3}$.The equivalent circuit corresponding to equation (33) is shown in Figure10a. This circuit is made up regarding voltage sources. According to known rules, it can be converted to the source of the current, Figure 10b, where

$$
g_{d}(k)=\left(r_{d}+j k x_{d}\right)^{-1}, \quad J_{d}(k)=\frac{\delta \dot{E}(k)-\frac{2 x_{d}}{\pi} \cdot \dot{a}(k) \cdot i_{d}(\alpha)}{r_{d}+j k x_{d}}
$$

Consider the extreme case when $x_{d} \rightarrow \infty$. 


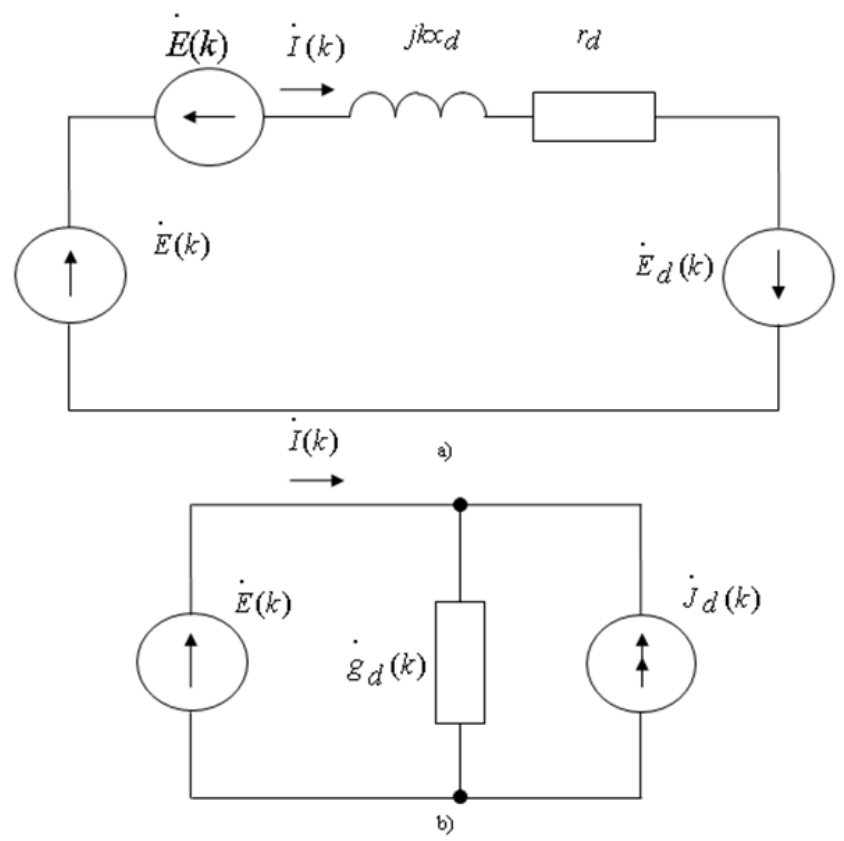

Figure 10. Equivalent circuit of the converter on the k-th harmonic

$$
\begin{gathered}
\text { a-with voltage sources } \dot{E}_{d}(k)=\frac{2}{\pi} x_{d} \dot{a}(k) i_{d}(\alpha) ; \\
\text { b-with current source } \dot{J}_{d}(k) .
\end{gathered}
$$

It is obvious then that $g_{d}(k) \rightarrow 0$ and, taking into account the chosen direction of the current source, the equation will be:

$$
\lim _{x_{d} \rightarrow \infty} J_{d}(k)=\frac{2 j}{\pi k} a(k) i_{d}(\alpha)=\frac{2 j I_{d}}{\pi k} a(k)
$$

The resulting equation coincides with the result of the decomposition on harmonic components completely smoothed on intervals of conduction $\theta \in[\alpha ; \alpha+2 \pi / 3], \quad \theta \in[\alpha+\pi ; \alpha+5 \pi / 3]$ phase current. Equation (34) and (35) allow for the correct substantiate methodologically, when the current source introduced in the equivalent circuit and when specifying the current, and what assumptions are implicitly used in this. In case of limiting translation to (35) we should waive the submission of the converter load in the form of the current source and move to the specified current. When we remove the uncertainty of the current source fault through the load conductance (formally at $g_{d}(k)=0$ voltage on the conduction equal $\infty$ ).It is known that in this case the load circuit in the voltages balance equation do not drop in, and the currents involved in the equations of the first Kirchhoff's law. Equation (35) explains, how the composition of current harmonics of rectifier load depends on the angle of the control: their relative content remains constant for any value of $\alpha$, and the amplitudes are directly proportional to the load current $i_{d}(\alpha) \equiv i_{d}(\theta)=I_{d}=$ const .

Let us now consider in more detail the equation (34) and write it in the following form: 


$$
\dot{E}(k)=\left(r_{d}+j k x_{d}\right) \cdot\left[I(k)+J_{d \delta}(k)+J_{d i}(k)\right],
$$

where

$$
J_{d \delta}(k)=\frac{\delta \dot{E}(k)}{r_{d}+j k x_{d}} ; \quad J_{d i}(k)=-\frac{2 x_{d} \cdot \dot{a}(k)}{\pi\left(r_{d}+j k x_{d}\right)} \cdot i_{d}(\alpha) .
$$

If the second term in equation (36) is written, first, as

$$
J_{*} d i(k)=\frac{J_{d i}(k)}{i_{d}(\alpha)}=\frac{2}{\pi} \cdot \frac{\dot{a}(k)}{\xi+j k}
$$

and, second, determine the modulus, we get that current harmonics module depends only on the ratio of the load parameters $\xi=r_{d} / x_{d}$ :

$$
J_{d i}(k)=\left|\begin{array}{c}
J_{d i}(k) \\
*
\end{array}\right|=\frac{2 \sqrt{3}}{\pi \sqrt{\xi^{2}+k^{2}}} .
$$

The first term in (36) is normalized to the ratio for the current $i_{d}(\alpha)$

$$
\underset{*}{J_{d \delta}}(k)=\frac{-\delta E(k)}{i_{d}(\alpha) \cdot\left(r_{d}+\mathrm{j} k x_{d}\right)} \Rightarrow \underset{*}{J_{d \delta}}(k)=\left|J_{*} \underset{*}{ }(k)\right|=\frac{|\delta E(k)|}{i_{d}(\alpha) \cdot x_{d} \sqrt{\xi^{2}+k^{2}}} .
$$

We write the equation for calculating the rectified current in the circuit of Figure 8 in the instantaneous values of variables (4):

$$
i_{d}(\theta)=\frac{\sqrt{3} E}{Z_{d}} \cos \left(\theta-\frac{\pi}{3}-\varphi_{d}\right)-\left[\frac{\sqrt{3} E}{Z_{d}} \cos \left(\alpha-\frac{\pi}{3}-\varphi_{d}\right)-i_{d}(\alpha)\right] e^{-(\theta-\alpha) \xi}
$$

In steady state operation

$$
i_{d}(\alpha+\pi / 3)=i_{d}(\alpha)
$$

Substituting the value $\theta=\alpha+\pi / 3$ in equation (39), we find

$$
i_{d}(\alpha)=\frac{\sqrt{3} E}{x_{d} \sqrt{\xi^{2}+1}} \frac{\cos \left(\alpha-\varphi_{d}\right)-\cos \left(\alpha-\pi / 3-\varphi_{d}\right) \cdot \mathrm{e}^{-\pi \cdot \xi / 3}}{1-\mathrm{e}^{-\pi \cdot \xi / 3}} .
$$

Now we substitute $i_{d}(\alpha)$ from equation (40) in equation (38):

$$
\underset{*}{J_{d} \delta}(k)=\frac{|\delta E(k)|}{\sqrt{3} E} \times \frac{1-\mathrm{e}^{-\pi \xi / 3}}{\cos \left(\alpha-\varphi_{d}\right)-\cos \left(\alpha-\pi / 3-\varphi_{d}\right) \cdot \mathrm{e}^{-\pi \xi / 3}} \times \frac{\sqrt{\xi^{2}+1}}{\sqrt{\xi^{2}+k^{2}}} .
$$

If we normalize the harmonics at the average rectified current $I_{d}=I_{d}(0)=\frac{3 \sqrt{3}}{\pi r_{d}} E \cos (\alpha-\pi / 6)$ then in equations (37) and (41) we must enter the correction coefficient $k_{i}=i_{d}(\alpha) / I_{d}$. Calculations show that $k_{i}=0.9384$ at $\chi=\xi^{-1}=0,1$ and $k_{i}=0.9959$ already at $\chi=1$ In Figure 11a we set up characteristics of the second term in (36)- current $J_{d i}=f(\xi, k)$ for different harmonics. 
$I_{d i}(k)$, o.e.

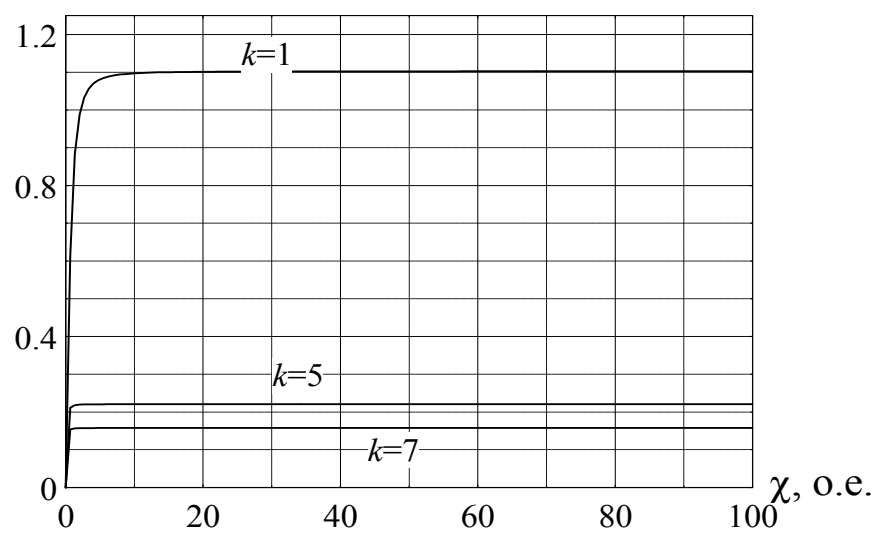

a)

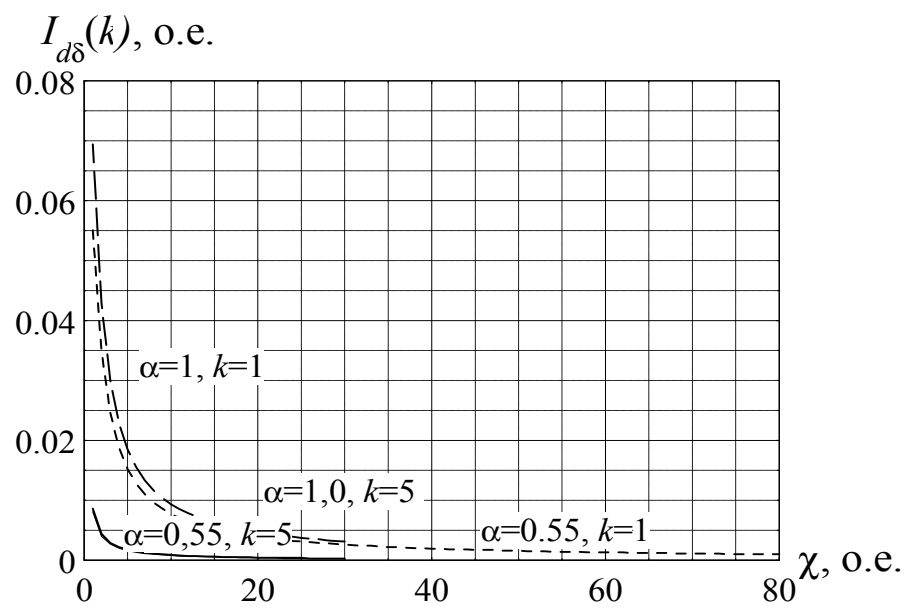

б)

Figure 11. Harmonic components of the rectified current a - harmonic current caused by the voltage source; $\sigma$ - harmonic current caused by EMF distortion.

In Figure $11 \mathrm{~b}$ we set up characteristics of the first term

$$
J_{d \delta}=f(\xi, k)
$$

for different harmonics.

Comparison of the graphs shows that an effect of less than $2 \%$ on the first harmonic provides the first term in equation (36) to the total current even at $\chi \geq 5$. For the other harmonics the same result occurs when $\chi \geq 1$. It can be concluded that the EMF distortion shows itself appreciably (more than 5\%) only at the ratios of active resistance and inductance of the rectifier load $\chi \leq 5$. In the other cases, we can ignore the effect of EMF distortion and assume it in the equivalent circuit in Figure 9 to be equal to zero.

2.6 Initial Equations Converter Relative to One Phase with Respect to Switching Processes

Let us consider the circuit shown in Figure 12. 


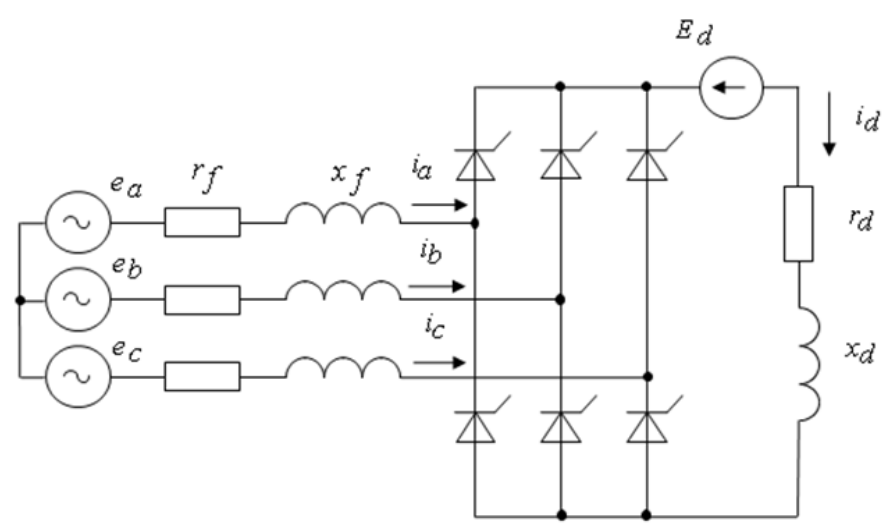

Figure 12. Circuit diagram converter and the mains supply

Steady state operation of the converter corresponds to the diagram of the phase currents, Figure 13.

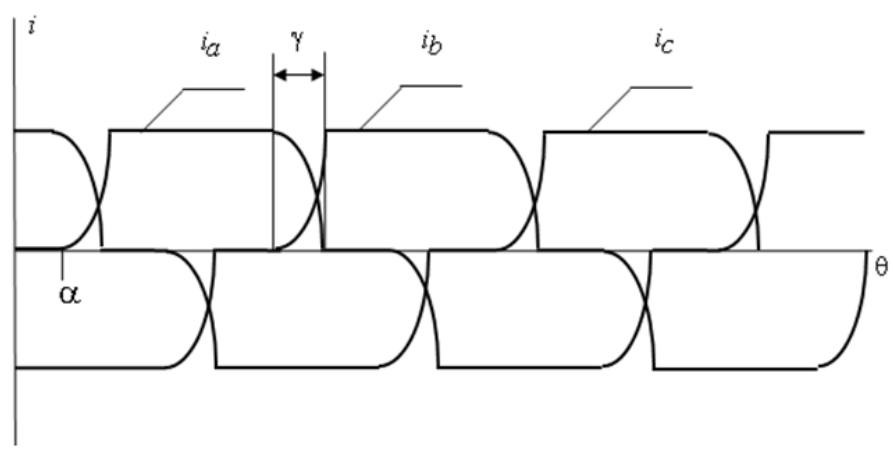

Figure 13. Diagram of phase currents converter

We write the balance equation voltages for the phase " $a$ " at the interval $[\alpha ; \alpha+2 \pi]$ considering the switching processes. Balance equations of the phase voltages "a" and "b" in the first half cycle:

$$
\left.\begin{array}{l}
e_{a}-e_{b}=r_{f} \cdot i_{a}+x_{f} \frac{d i_{a}}{d \theta}+\left(r_{f}+r_{d}\right) \cdot i_{d}+\left(x_{f}+x_{d}\right) \frac{d i_{d}}{d \theta}+E_{d}, \\
\quad \theta \in[\alpha ; \alpha+\gamma] ; \\
e_{a}-e_{b}=r_{S} \cdot i_{a}+x_{S} \frac{d i_{a}}{d \theta}+E_{d}, \\
\theta \in[\alpha+\gamma ; \alpha+\pi / 3] ; r_{S}=2 r_{f}+r_{d} ; x_{S}=2 x_{f}+x_{d} .
\end{array}\right\}
$$

Balance equations of the phase voltages "a" and "c" in the first half cycle:

$$
\left.\begin{array}{l}
e_{a}-e_{c}=\left(r_{f}+r_{d}\right) \cdot i_{a}+\left(x_{f}+x_{d}\right) \frac{d i_{a}}{d \theta}-r_{f} \cdot i_{c}-x_{f} \frac{d i_{c}}{d \theta}+E_{d}, \\
\quad \theta \in[\alpha+\pi / 3 ; \alpha+\pi / 3+\gamma] ; \\
e_{a}-e_{c}=r_{S} \cdot i_{a}+x_{S} \frac{d i_{a}}{d \theta}+E_{d}, \quad \theta \in[\alpha+\pi / 3+\gamma ; \alpha+2 \pi / 3] .
\end{array}\right\}
$$

Balance equations of the phase voltages "a" and "b" in the second half cycle: 


$$
\left.\begin{array}{l}
e_{a}-e_{b}=r_{f} \cdot i_{a}+x_{f} \frac{d i_{a}}{d \theta}-\left(r_{f}+r_{d}\right) \cdot i_{d}-\left(x_{f}+x_{d}\right) \frac{d i_{d}}{d \theta}-E_{d}, \\
\theta \in[\alpha+\pi ; \alpha+\pi+\gamma] ; \\
e_{a}-e_{b}=r_{s} \cdot i_{a}+x_{S} \frac{d i_{a}}{d \theta}-E_{d}, \quad \theta \in[\alpha+\pi+\gamma ; \alpha+4 \pi / 3] .
\end{array}\right\}
$$

Balance equations of the phase voltages "a" and "c" in the second half cycle:

$$
\left.\begin{array}{c}
e_{a}-e_{c}=\left(r_{f}+r_{d}\right) \cdot i_{a}+\left(x_{f}+x_{d}\right) \frac{d i_{a}}{d \theta}-r_{f} \cdot i_{c}-x_{f} \frac{d i_{c}}{d \theta}-E_{d}, \\
\theta \in[\alpha+5 \pi / 3 ; \alpha+5 \pi / 3+\gamma] ; \\
e_{a}-e_{c}=r_{s} \cdot i_{a}+x_{s} \frac{d i_{a}}{d \theta}-E_{d}, \quad \theta \in[\alpha+\pi / 3+\gamma+\pi ; \alpha+5 \pi / 3] .
\end{array}\right\}
$$

We introduce the switching currents:

- $\quad$ at the interval $\theta \in[\alpha ; \alpha+\gamma] \quad i_{\gamma 1}=i_{d}-i_{a} \Rightarrow i_{\gamma 1}(\alpha)=i_{d}(\alpha) ; \quad i_{\gamma 1}(\alpha+\gamma)=0$

- $\quad$ at the interval $\theta \in[\alpha+\pi / 3 ; \alpha+\pi / 3+\gamma]: \quad i_{\gamma 2}=-i_{c}-i_{a} \Rightarrow$

$$
\Rightarrow i_{\gamma 2}(\alpha+\pi / 3)=-i_{\mathrm{a}}(\alpha+\pi / 3)=-i_{d}(\alpha+\pi / 3)=-i_{d}(\alpha) ; i_{\gamma 2}(\alpha+\pi / 3+\gamma)=0 ;
$$

- $\quad$ at the interval $\theta \in[\alpha+\pi ; \alpha+\gamma+\pi]$

$i_{\gamma 3}=-i_{d}-i_{a} \Rightarrow i_{\gamma 3}(\alpha+\pi)=-i_{d}(\alpha+\pi)=-i_{d}(\alpha) ; \quad i_{\gamma 3}(\alpha+\gamma+\pi)=0 ;$

- $\quad$ at the interval $\theta \in[\alpha+5 \pi / 3 ; \alpha+5 \pi / 3+\gamma]: \quad i_{\gamma 4}=-i_{a}-i_{c} \Rightarrow$

$\Rightarrow i_{\gamma 4}(\alpha+5 \pi / 3)=-i_{a}(\alpha+5 \pi / 3)=i_{d}(\alpha+5 \pi / 3)=i_{d}(\alpha) ; i_{\gamma 4}(\alpha+5 \pi / 3+\gamma)=0$.

We use substitutions for the currents:

$$
\begin{aligned}
& i_{d}=i_{a}+i_{\gamma 1}, \theta \in[\alpha ; \alpha+\gamma] ; \quad-i_{c}=i_{a}+i_{\gamma 2}, \quad \theta \in[\alpha+\pi / 3 ; \alpha+\pi / 3+\gamma] \\
& -i_{d}=i_{a}+i_{\gamma 3}, \theta \in[\alpha+\pi ; \alpha+\gamma+\pi] ;-i_{c}=i_{a}+i_{\gamma 4}, \theta \in[\alpha+5 \pi / 3 ; \alpha+5 \pi / 3+\gamma] .
\end{aligned}
$$

Accordingly, equations (42) - (45) take the following form:

$$
\left.\begin{array}{r}
e_{a}-e_{b}=r_{S} i_{a}+x_{S} \frac{d i_{a}}{d \theta}+\left(r_{f}+r_{d}\right) i_{\gamma 1}+\left(x_{f}+x_{d}\right) \frac{d i_{\gamma 1}}{d \theta}+E_{d}, \quad \theta \in[\alpha ; \alpha+\gamma] ; \\
e_{a}-e_{b}=r_{S} i_{a}+x_{s} \frac{d i_{a}}{d \theta}+E_{d}, \quad \theta \in[\alpha+\gamma ; \alpha+\pi / 3] . \\
e_{a}-e_{c}=r_{S} i_{a}+x_{s} \frac{d i_{a}}{d \theta}+r_{f} i_{\gamma 2}+x_{f} \frac{d i_{\gamma 2}}{d \theta}+E_{d}, \theta \in[\alpha+\pi / 3 ; \alpha+\pi / 3+\gamma] ; \\
e_{a}-e_{c}=r_{S} i_{a}+x_{s} \frac{d i_{a}}{d \theta}+E_{d}, \quad \theta \in[\alpha+\pi / 3+\gamma ; \alpha+2 \pi / 3] . \\
e_{a}-e_{b}=r_{S} \cdot i_{a}+x_{S} \frac{d i_{a}}{d \theta}+\left(r_{f}+r_{d}\right) \cdot i_{\gamma 3}+\left(x_{f}+x_{d}\right) \frac{d i_{\gamma 3}}{d \theta}-E_{d}, \theta \in[\alpha+\pi ; \alpha+\pi+\gamma] ; \\
e_{a}-e_{b}=r_{S} \cdot i_{a}+x_{s} \frac{d i_{a}}{d \theta}-E_{d}, \quad \theta \in[\alpha+\pi+\gamma ; \alpha+\pi+\pi / 3] .
\end{array}\right\}
$$




$$
\left.\begin{array}{l}
e_{a}-e_{c}=r_{S} i_{a}+x_{S} \frac{d i_{a}}{d \theta}+r_{f} i_{\gamma 4}+x_{f} \frac{d i_{\gamma 4}}{d \theta}-E_{d}, \theta \in[\alpha+5 \pi / 3 ; \alpha+5 \pi / 3+\gamma] ; \\
e_{a}-e_{c}=r_{S} i_{a}+x_{S} \frac{d i_{a}}{d \theta}-E_{d}, \quad \theta \in[\alpha+4 \pi / 3+\gamma ; \alpha+5 \pi / 3] .
\end{array}\right\}
$$

We combine voltage balance equations (46) - (49):

$$
\begin{aligned}
& \mathrm{e}_{a}=\left(r_{s} i_{a}+x_{s} \frac{d i_{a}}{d \theta}\right) K_{a}(\theta)+\left[\left(r_{f}+r_{d}\right) i_{\gamma 1}+\left(x_{f}+x_{d}\right) \frac{d i_{\gamma 1}}{d \theta}\right] K_{\gamma 1}(\theta)+ \\
& +\left(r_{f} i_{\gamma 2}+x_{f} \frac{d i_{\gamma 2}}{d \theta}\right) K_{\gamma 2}(\theta)+\left[\left(r_{f}+r_{d}\right) i_{\gamma 3}+\left(x_{f}+x_{d}\right) \frac{d i_{\gamma 3}}{d \theta}\right] K_{\gamma 3}(\theta)+ \\
& +\left(r_{f} i_{\gamma 4}+x_{f} \frac{d i_{\gamma 4}}{d \theta}\right) K_{\gamma 4}(\theta)+\delta e_{a} .
\end{aligned}
$$

Figure 14 shows plots of the switching currents, and Figure 15 - key (switching) functions $K(\theta)$.

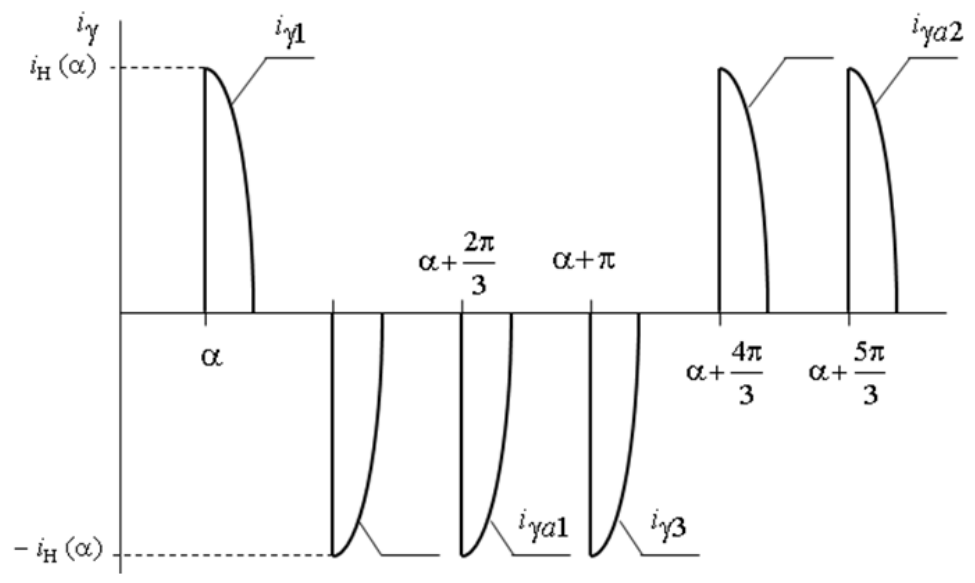

Figure 14. Switching currents of phase " $a$ "

Equation (50) needs to be supplemented, as a function not cover the entire conduction interval of phase "a", which will lead to an incorrect calculation of the phase current harmonics. Therefore, to account for the switching intervals, corresponding to the transition the current of phase "a" to phase «b», we introduce on the right side of equation (50) the terms:

$$
\pm\left(r_{S} \cdot i_{a}+x_{S} \frac{d i_{a}}{d \theta}\right) \cdot K_{\gamma a 1}(\theta)
$$

and

$$
\pm\left(r_{S} \cdot i_{a}+x_{S} \frac{d i_{a}}{d \theta}\right) \cdot K_{\gamma a 2}(\theta)
$$

then we get 


$$
\begin{aligned}
& e_{a}=\left(r_{s} i_{a}+x_{S} \frac{d i_{a}}{d \theta}\right) K_{S}(\theta)+\left[\left(r_{f}+r_{d}\right) i_{\gamma 1}+\left(x_{f}+x_{d}\right) \frac{d i_{\gamma 1}}{d \theta}\right] K_{\gamma 1}(\theta)+ \\
& +\left(r_{f} i_{\gamma 2}+x_{f} \frac{d i_{\gamma 2}}{d \theta}\right) K_{\gamma 2}(\theta)+\left(r_{s} i_{\gamma a 1}+x_{S} \frac{d \dot{j}_{\gamma a 1}}{d \theta}\right) K_{\gamma a 1}(\theta)+ \\
& +\left[\left(r_{f}+r_{d}\right) i_{\gamma 3}+\left(x_{f}+x_{d}\right) \frac{d i_{\gamma 3}}{d \theta}\right] K_{\gamma 3}(\theta)+\left(r_{f} i_{\gamma 4}+x_{f} \frac{d i_{\gamma 4}}{d \theta}\right) K_{\gamma 4}(\theta)+ \\
& +\left(r_{s} i_{\gamma a 2}+x_{S} \frac{d i_{\gamma a 2}}{d \theta}\right) K_{\gamma a 2}(\theta)+\delta e_{a} .
\end{aligned}
$$

Where the following boundary conditions are fulfilled:

$$
i_{\gamma a 1}=-i_{a} \text { where }^{\theta \in[\alpha+2 \pi / 3 ; \alpha+2 \pi / 3+\gamma]} \text { and } i_{\gamma a 2}=-i_{a} \text { at } \theta \in[\alpha+5 \pi / 3 ; \alpha+5 \pi / 3+\gamma] .
$$

Thus we get equation (51), which reduces the rectifying process of a three-phase circuit to mode with respect to a single phase.

2.7 Equivalent Circuit of the Three-Phase Thyristor Converter, Taking into Account the Switching Processes We apply the expression (51) LFT. At the same time we take into account that $i_{a}=0$ at the boundaries of the key function $K_{S}(\theta)$.In result (Figure 15) we get:

$$
\begin{aligned}
& \dot{E}_{a}(k)=\left(r_{S}+j k x_{S}\right) \dot{I}_{a}(k)+\left(r_{S}+j k x_{S}\right) \dot{I_{\gamma a}}(k)+ \\
& +\left[\left(r_{f}+r_{d}\right)+j k\left(x_{f}+x_{d}\right)\right] \dot{I} \dot{I}_{\gamma 13}(k)+\left(r_{f}+j k x_{f}\right) \dot{I} \gamma 24(k)-\frac{x_{f}+x_{d}}{\pi} i_{d}(\alpha)+ \\
& +\frac{x_{f}+x_{d}}{\pi} \dot{I}_{d}(\alpha) e^{-j k \pi}+\frac{x_{f}}{\pi} i_{d}(\alpha) e^{-j k \pi}-\frac{x_{f}}{\pi} i_{d}(\alpha) e^{-j k(\pi+\pi / 3)}+ \\
& +\frac{x_{S}}{\pi} i_{d}(\alpha) e^{-j k 2 \pi / 3}-\frac{x_{S}}{\pi} i_{d}(\alpha) e^{-j k(\pi+2 \pi / 3)}+\delta \dot{E}(k) .
\end{aligned}
$$

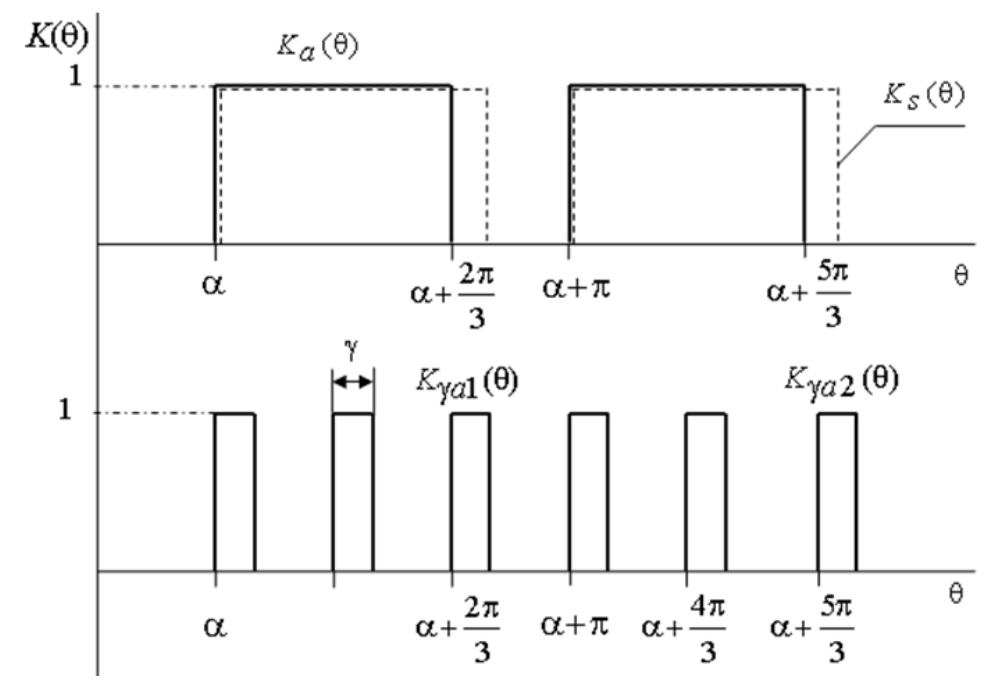

Figure 15. Switching functions

In equation (52) we use the following notation for the complex amplitudes of the harmonics of switching currents: 


$$
\begin{gathered}
I_{\gamma 13}(k)=\frac{1}{\pi} \int_{\alpha}^{\alpha+\gamma} i_{\gamma 1} \cdot e^{-j k(\theta-\alpha)} d \theta+\frac{1}{\pi} \int_{\alpha+\pi}^{\alpha+\gamma+\pi} i_{\gamma 3} \cdot e^{-j k(\theta-\alpha)} d \theta ; \\
\dot{I}_{\gamma 24}(k)=\frac{1}{\pi} \int_{\alpha+\pi / 3}^{\alpha+\pi / 3+\gamma} i_{\gamma 2} \cdot e^{-j k(\theta-\alpha)} d \theta+\frac{1}{\pi} \int_{\alpha+4 \pi / 3}^{\alpha+4 \pi / 3+\gamma} i_{\gamma 4} \cdot e^{-j k(\theta-\alpha)} d \theta ; \\
\dot{I}_{\gamma a}(k)=\frac{1}{\pi} \int_{\alpha+2 \pi / 3}^{\alpha+2 \pi / 3+\gamma} i_{\gamma a 1} \cdot e^{-j k(\theta-\alpha)} d \theta+\frac{1}{\pi} \int_{\alpha+5 \pi / 3}^{\alpha+5 \pi / 3+\gamma} i_{\gamma 2} \cdot e^{-j k(\theta-\alpha)} d \theta
\end{gathered}
$$

We take into account that for switching currents there are the following boundary conditions:

$$
\begin{aligned}
& i_{\gamma a 1}(\alpha+\gamma+2 \pi / 3)=i_{\gamma a 2}(\alpha+\gamma+5 \pi / 3)=i_{\gamma 1}(\alpha+\gamma)=i_{\gamma 2}(\alpha+\gamma+\pi / 3)= \\
& =i_{\gamma 3}(\alpha+\pi+\gamma)=i_{\gamma 4}(\alpha+4 \pi / 3+\gamma)=0 \\
& \quad i_{\gamma a 1}(\alpha+2 \pi / 3)=i_{\gamma 2}(\alpha+\pi / 3)=i_{\gamma 3}(\alpha+\pi)=-i_{\mathrm{H}}(\alpha) ; \\
& i_{\gamma 1}(\alpha)=i_{\gamma 4}(\alpha+4 \pi / 3)=i_{\gamma a 2}(\alpha+5 \pi / 3)=i_{\mathrm{H}}(\alpha) .
\end{aligned}
$$

Taking into account that in the equation (53) the number of harmonics $k$ takes only odd values and multiples of three. Then, omitting the index of phase, we have:

$$
\begin{aligned}
& \dot{E}(k)=\left(r_{S}+j k x_{S}\right) \dot{I}(k)+\delta \dot{E}(k)+\left(r_{S}+j k x_{S}\right) \dot{J_{\gamma a}}(k)+ \\
& +\left[\left(r_{f}+r_{d}\right)+j k \cdot\left(x_{f}+x_{d}\right)\right] \cdot J_{\gamma 13}(k)+\left(r_{f}+j k x_{f}\right) \cdot J_{\gamma 24}(k)- \\
& -\left[\frac{2}{\pi} x_{S}\left(1-e^{-j k 2 \pi / 3}\right)-\frac{2}{\pi} x_{f}\left(1+e^{-j k \pi / 3}\right)\right] \cdot i_{d}(\alpha) .
\end{aligned}
$$

In accordance with equation (53) in Figure 16 we present the equivalent circuit of a three-phase converter, reduced to one phase, where we use the notation:

$$
\begin{aligned}
& \dot{J}_{1}(k)=\dot{J}_{\gamma 24}(k), \dot{J}_{2}(k)=\dot{J}_{\gamma 13}(k), \dot{J}_{3}(k)=\dot{J}_{\gamma a}(k), r_{S}^{\prime}=r_{f}+r_{d} ; x_{s}^{\prime}=x_{f}+x_{d} ; \\
& \dot{E}_{d}(k)=\left[\frac{2}{\pi} x_{S}\left(1-e^{-j k 2 \pi / 3}\right)-\frac{2}{\pi} x_{f}\left(1+e^{-j k \pi / 3}\right)\right] \cdot i_{d}(\alpha) .
\end{aligned}
$$

It is easy to see that for $x_{d} \rightarrow \infty$ we have

$$
\lim _{x_{d} \rightarrow \infty} \dot{I}(k)=\frac{2}{\pi j k} \dot{a}(k) i_{d}(\alpha)-J_{2}(k)-J_{3}(k)=\frac{2 I_{d}}{\pi j k} \dot{a}(k)-J_{2}(k)-J_{3}(k)
$$

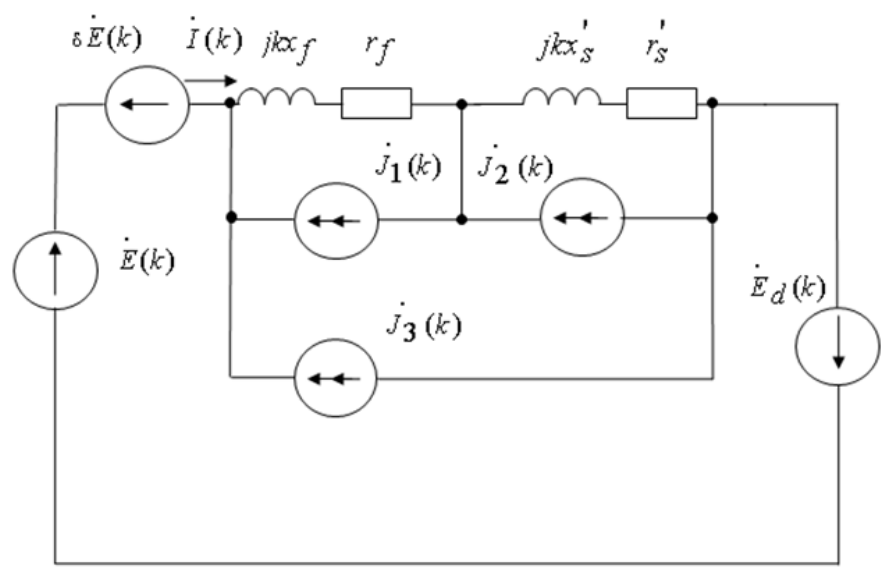

Figure 16. Equivalent circuit of three-phase thyristor converter for calculating current and voltage harmonics 


\section{Results}

Formula (54) is none other than a complex spectrum of the phase current at the input of the thyristor converter, when the rectified current is completely flattened. If phase EMF are sinusoidal, then analogous results were obtained in (16), when harmonic currents are decomposed into sine and cosine components. On the basis of the mathematical apparatus of the local Fourier transform we receive the equivalent circuit of a three-phase manage thyristor converter, allowing use to calculate harmonic currents in the power supply system. It is proved that without switching the equivalent circuit reduces to a voltage source and impedance. The influence of the network on the equivalent circuit has an effect through the angle control, which depends on the voltage supply for a given average rectified current, whose value is determined by technological process and maintained by the control system unchanged. When accounting for switching processes in the equivalent circuit, three current sources also enter, caused by passage of switching currents. As a result, the calculation of harmonic currents in the power supply system and associated losses of power occurs in two stages: initially the operation regime of the power supply system is calculated assuming the rated voltage on thyristor converters, and then the switching currents and control angles are adjusted, and then there is second recalculation of the harmonics.

\section{Discussion}

1. The resulting mathematical model of single-phase and three-phase converters include as the main element of their resistive and inductive loads.

2. Peculiarity of the mathematical model is a combination of a current source and a voltage source in the equivalent circuit. Parameters of the current source depend from the number of higher harmonic and laws of change the feeding phase currents during commutation thyristors. Parameters of the voltage source depend only from the inductance of the load and the load current, but are independent of the number of harmonics of the phase current.

3. Changing the harmonic content of the phase currents in each line of activity in the AC occurs in the interaction of several controlled thyristor converters through the bus of common coupling.

4. Process of mutual influence of converters is in changing the commutation processes of each of them under the influence of a changing spectrum of voltage harmonics in the current of common bus in group work of thyristor converters.

5. Mutual influence of thyristor converters is present as a change in the parameters of harmonic current sources presented in equivalent circuits. Revealed features show the necessity of rejecting away from assumptions about the sinusoidal variation of the current switching in group work of thyristor converters. Generally illustrates the impossibility of using the principle of superposition of harmonic currents and voltages, calculated by a conventional method.

\section{Conclusion}

This paper have indicated a principle of superposition of harmonic currents can not be extrapolated to the case of group work thyristor converters. The reason is that higher harmonics in the spectrum of each line acceding unchanged compared with an individual in this mode of operation the mains inverter unit. This paper should be addressed by researchers on power-system harmonics. The present list of research challenges is most likely not complete, but the authors hope that it will give some guidance to the future research on power-system. In the future, we plan to create a mathematical algorithm of thyristor converter. On the basis of a mathematical algorithm of the thyristor converter will be set up software to protect of higher harmonics. Software-based microelectronic device will be created to prevent the higher harmonics.

\section{Acknowledgments}

The presented results had obtained through scientific research work «Methods of increase of reliability and quality of electric power supply in the distribution networks» (the task number 2014/448 on the implementation of government works in the field of scientific activities of the base portion of the state task of the Ministry of Education and Science of Russia).

\section{References}

Alexander, K., Sc.D., P. E., \& Marc, T. T. (2007). Power Quality in Electrical Systems. McGraw-Hill Companies, Inc.

Andresen, B. et al. (2012). Decomposition and mitigation of a disturbance being present at an electric connection between an electric power generating system and a power grid, Europe Patent EP2630510 A1. 
Code of Federal Regulation. Title 47. Part 15. Subpart J. Computing Devices.

Curatolo, T., \& Cogger, S. (2005). Enhancing a Power Supply to Ensure EMI Compliance. EDN, pp. 67-74.

Fedotov, A. (1999). The discrete operator method of calculation of transient processes in electrical circuits with a rectifier load. Electrotechnics, 3.

Fedotov, A. (2001). The calculation of transient processes in synchronous machines with independent thyristor excitation by discrete operational method. Electricity, 5 .

Fedotov, A. I. et al. (2003). Theoretical bases of discrete simulation electromachines-valve systems. Scientific publication. Kazan Univ KSPEU.

Gallo, D. et al. (2004). IEC Flickermeter Response to Interharmonic Pollution. International Conference on Harmonics and Quality of Power, 12 - 15, 489-494.

Harmonics in electrical systems (1990). Trans. from English. Arrilaga J. etc. M. Energoatomizdat.

IEEE Recommended Practice for Measurement and Limits of Voltage Fluctuations and Associated Light Flicker on AC Power Systems. IEEE St. 1453- 2004.

Jan, M. et al. (2014). Future Work on harmonics - Some Expert Opinions. Part II - Supraharmonics, Standards and Measurements. 16th IEEE International Conference on Harmonics and Quality of Power, Proceedings,.

Kuznetsov, V., \& Fedotov, A. (1999). The use of the local Fourier transformation for the mathematical modeling of synchronous machines with thyristor excitation systems. Electricity, 4.

Math, B. et al. (2014). Future Work on Harmonics - Some Expert Opinions. Part I - Wind and Solar Power. 16th IEEE International Conference on Harmonics and Quality of Power, Proceedings.

Sermon, R. C. (2005). An Overview of Power Quality Standards and Guidelines from the End-User's Point-of-View. Proceedings of the 2005 Rural Electric Power Conference, pp. B1-1-B1-5.

Takeuchi, T. (1973). The theory and application of thyristor circuits for motor control. Trans. from English. Leningrad: Energy.

Tolstov, J., \& Tevryukov, A. (1971). The theory of electrical circuits. Moscow: High. Sch.

Vicaschev, A., \& Tiguntsev, S. (2002). The influence of consumers on the voltage distortion. Electric Power Plants, 7 .

Wang, C. S., \& Devaney, M. J. (2004). Incandescent Lamp Flicker Mitigation and Measurement. IEEE Transactions on Instrumentation and Measurement, 53(4), 1028-1034.

Zhezhelenko, I. V. (1994). Higher harmonics in power systems industrial plants. 3rd ed. Energoatomizdat.

ГОСТ P 54149-2010. Electric Energy. Electromagnetic Compatibility of Technical Equipment. Power Quality Limits in Public Electrical System. Retrieved from $\mathrm{http} / /$ protect.gost.ru/nom.aspx mode $=$ doc\&id=132893\&nomentype $=1 \&$ baseC $=28 \&$ page $=0 \&$ code $=01$

\section{Copyrights}

Copyright for this article is retained by the author(s), with first publication rights granted to the journal.

This is an open-access article distributed under the terms and conditions of the Creative Commons Attribution license (http://creativecommons.org/licenses/by/3.0/). 